\title{
IMPROVEMENTS AND COMPARISON OF HEURISTICS FOR SOLVING THE UNCAPACITATED MULTISOURCE WEBER PROBLEM
}

\author{
JACK BRIMBERG \\ School of Business Administration, University of Prince Edward Island, Charlottetown, Canada C1A 4P3, jbrimberg@upei.ca \\ PIERRE HANSEN and NENAD MLADENOVIĆ \\ GERAD and Ecole des Hautes Etudes Commerciales, 3000, chemin de la Côte-Sainte-Catherine, Montreal, Canada H3T $2 A 7$ \\ pierreh@crt.umontreal.ca・nenad@crt.umontreal.ca
}

ERIC D. TAILLARD

IDSIA, Corso Elvezia 36,CH-6900 Lugano, Switzerland, eric.taillard@eivd.ch

(Received June 1997; revision received May 1998; accepted July 1998)

\begin{abstract}
The multisource Weber problem is to locate simultaneously $m$ facilities in the Euclidean plane to minimize the total transportation cost for satisfying the demand of $n$ fixed users, each supplied from its closest facility. Many heuristics have been proposed for this problem, as well as a few exact algorithms. Heuristics are needed to solve quickly large problems and to provide good initial solutions for exact algorithms. We compare various heuristics, i.e., alternative location-allocation (Cooper 1964), projection (Bongartz et al. 1994), Tabu search (Brimberg and Mladenović 1996a), p-Median plus Weber (Hansen et al. 1996), Genetic search and several versions of Variable Neighbourhood search. Based on empirical tests that are reported, it is found that most traditional and some recent heuristics give poor results when the number of facilities to locate is large and that Variable Neighbourhood search gives consistently best results, on average, in moderate computing time.
\end{abstract}

\section{INTRODUCTION}

The multisource Weber problem (also known as the location-allocation problem) requires locating a set of facilities and simultaneously allocating to these facilities demands for service from a set of customers in order to optimize some performance criterion. This problem occurs in many practical settings where facilities provide a homogeneous service, such as the location of plants, warehouses, retail outlets, and public facilities. In the continuous version of the location-allocation problem, referred to as the multisource Weber problem, the objective is to generate $m$ new facility sites in $\mathbb{R}^{2}$ to serve the demands of $n$ customers or fixed points in such a manner as to minimize the total transportation (or service) cost. The uncapacitated version we consider may be formulated as follows (e.g., see Love et al. 1988):

$$
\begin{array}{ll}
\min _{W, X} & \sum_{i=1}^{n} \sum_{j=1}^{m} w_{i j}\left\|x_{j}-a_{i}\right\| \\
\text { s.t. } & \sum_{j=1}^{m} w_{i j}=w_{i}, \quad i=1, \ldots, n, \\
& w_{i j} \geqslant 0, \quad \forall i, j,
\end{array}
$$

where

$a_{i}=\left(a_{i 1}, a_{i 2}\right)$ is the known location of customer $i, i=1, \ldots, n$;
$X=\left(x_{1}, \ldots, x_{m}\right)$ denotes the matrix of location decision variables, with $x_{j}=\left(x_{j 1}, x_{j 2}\right)$ being the unknown location of facility $j, j=1, \ldots, m$;

$w_{i}$ is the given total demand or flow required by customer $i, i=1, \ldots, n$;

$W=\left(w_{i j}\right)$ denotes the vector of allocation decision variables, where $w_{i j}$ gives the flow to customer $i$ from facility $j, i=1, \ldots, n, j=1, \ldots, m$;

$\left\|x_{j}-a_{i}\right\|=\left[\left(x_{j 1}-a_{i 1}\right)^{2}+\left(x_{j 2}-a_{i 2}\right)^{2}\right]^{1 / 2}$ is the Euclidean norm.

The objective function above gives the total transportation cost, while the constraint set ensures that all customer demands are satisfied. Because there are no capacity constraints on the facilities, an optimal solution will have the demand at each customer served by the facility that is closest to it (ties being broken arbitrarily).

The main difficulty in solving (1) arises from the fact that the objective function is nonconvex (Cooper 1967) and, in general, contains a large number of local minima. Consider for example the well-known 50 customer problem in Eilon et al. (1971). Using 200 randomly generated starting solutions, the authors obtained 61 local minima for $m=5$, where the worst solution deviated from the best by $40.9 \%$. It was later shown (Krau 1997) that the best solution was indeed the global optimum. Thus, because of the complex shape of the objective function, the problem falls in the realm of global optimization. The problem may also be viewed as an enumeration of the Voronoi partitions of

Subject classifications: Facilities/equipment planning, location, continuous; heuristic solution methods. Area of review: TRANSPORTATION. 
the customer set and is known to be NP-hard (Megiddo and Supowit 1984).

As a consequence, exact methods for solving the locationallocation problem have been restricted until very recently to relatively small instances. Kuenne and Soland (1972) derive branch-and-bound algorithms that allow the solution of problem sizes of the order of 30 customers and 2 facilities or 15 customers and up to 4 facilities. The set reduction method and $p$-Median algorithm of Love and Morris (1975) for rectangular distances is restricted to similarly sized problems. An efficient solution procedure is developed for the special case of Euclidean distances and $m=2$ (Ostresh 1973a, Drezner 1984). This method is based on an observation that the subsets of customer locations allocated to the two facilities are separated by a straight line (Ostresh 1975). Computation times for up to 100 customers were reported at less than 14 seconds on an Amdahl 470/V8 computer (Drezner 1984). However, because there are $O\left(n^{2}\right)$ single facility location problems to solve, computation time will increase rapidly with $n$. More recently, Chen et al. (1992) have used a $d .-c$. programming method to solve the two-facility case more efficiently. The authors observe a near-linear increase in the computation time as $n$ increases and conclude that problem sizes of up to 1000 customers can be solved exactly. However, for three or more new facilities, the memory requirements of the method quickly restrict the problem sizes that can be attempted. A branchand-bound algorithm of Ostresh (1973b) solves problems with 3 facilities and 50 customers. Rosing (1992) extends the approach of Ostresh (1973a) in the following way: For a given number of facilities, potential subsets of customers served by the same facility (in other words, market areas) are determined by separating iteratively their set of locations by straight lines $n-1$ times. Then a large partitioning problem is solved, where each customer must belong to exactly one of those subsets. Unfortunately, the number of subsets augments very rapidly, and hence problems with 30 customers and 5 facilities or 25 customers and 6 facilities are typical of the size that can be solved.

The use of new tools has drastically augmented the size of problems that can be solved exactly. Krau (1997) proposes a column generation approach, combined with global optimization and branch-and-bound, which leads to the exact solution of instances with 287 customers and 2 to 100 facilities. These solutions are used for comparison of heuristics later in the paper. Combining this approach with a bundle method in the $l_{1}$-norm (du Merle et al. 1997) to stabilize solution of the dual leads to a very effective algorithm (Hansen et al. 1997) that can solve problems of up to 1000 customers and 100 facilities. To work well, both the column generation and the $l_{1}$-norm bundle method require an initial solution quite close to the optimum. Therefore, they use in an initial step the best heuristics developed in the present paper. Conversely, they provide exact solutions as a benchmark for large instances studied in this paper. Knowledge of exact optimal values (or tight bounds) is important, as some heuristics give solutions very far from the best values obtained by other ones (i.e., $100 \%$ or more for large $m$ ); and if optimal values are unknown, one might wonder if all heuristics give bad solutions for large instances or not. As will be shown below, this is not the case.

In the context of the multisource Weber problem, large problem sizes refer to the existence of multiple local optima, which make the global optimal solution difficult (if not impossible) to find in a reasonable amount of computing time. Furthermore, the complexity of the problem depends in a nonlinear manner on the parameters $n$ and $m$. Thus, a problem with as few as 50 customers may be relatively difficult to solve when the number of facilities is augmented to 25 . In the location-allocation literature, significantly larger problem sizes than this have been reported for the purpose of testing and comparison of alternative solution methods. Typically, these problems are generated in some random fashion. However, large-scale multisource Weber problems are also reported in practical applications with real data; e.g., consider the transshipment center location problem with $(m, n)$ of the order of $(20,1700)$ in Bhaskaran (1992) and the districting problem with $(m, n)$ of the order of $(170,1400)$ in Fleischmann and Paraschis (1988).

For the initialization of exact algorithms as well as for the approximate solution of very large problem instances that may occur in practice in terms of both parameters $n$ and $m$, heuristic methods are required. Many such methods have been proposed in the literature, beginning with the well-known iterative location-allocation algorithm of Cooper (1964). This heuristic uses the property that the location and allocation phases of the problem are very easy to solve in isolation. Thus, given the facility locations, each customer is simply allocated to its nearest one. Alternatively, knowing the allocation of the customers among the facilities, the problem reduces to the solution of $m$ independent single facility minisum problems, which because of the convexity of the objective function for normed distances are readily solved by descent methods such as the Weiszfeld procedure or variants thereof (e.g., see Kuhn 1973, Rosen and Xue 1991, Brimberg and Love 1993, Drezner 1992, Frenk et al. 1994, and Brimberg et al. 1996). Starting with an initial partition of the customer set, the Cooper algorithm alternates between the location and allocation phases until no further improvement can be made. Each iteration produces a lower value of the objective function until the process becomes trapped at a local minimum. A multistart version involves repeating the Cooper algorithm many times from randomly generated initial solutions and retaining the best local minimum from the trials as the final solution. Variants of the Cooper algorithm are discussed in Scott (1970) and Baxter (1981), while Sullivan and Peters (1980) propose a method to cluster customers into mutually exclusive subsets, in each of which a facility is then located.

Love and Juel (1982) devise a heuristic method with a defined neighbourhood structure. This neighbourhood consists of all the points around a current solution, which are obtained by exchanging a specified number of assignments of customers from their current facilities to new ones. Five 
variants of the proposed method are investigated. The first three algorithms, denoted as $\mathrm{H} 1$ to $\mathrm{H} 3$, use a single exchange, while the last two, $\mathrm{H} 4$ and $\mathrm{H} 5$, allow up to two exchanges. Different strategies such as first improvement and best improvement are employed to make descent moves from the current solution to a neighbourhood point. Again, because the search is local, the $\mathrm{H}$ heuristics of Love and Juel are guaranteed only to obtain a local minimum. The motivation for the larger neighbourhood of $\mathrm{H} 4$ and $\mathrm{H} 5$ is to better enable the algorithm to jump out of a "local optimum trap," but obviously, this comes at a large cost in computation time.

A completely different heuristic approach is given by Chen (1983). Using an approximation by Charalambous and Bandler (1976), the objective function is transformed by giving an exponent $(-N)$ to all distances between customers and facilities and an exponent $(-1 / N)$ to the sum of so modified distances from all facilities of each user. For $N$ sufficiently large, this last quantity approaches the distance between the customer and its closest facility. In this way, the allocation decision variables are eliminated. The resulting problem is then solved by the Broyden-Fletcher-Shanno quasi-Newton method (e.g., Avriel 1976). Good results, but not always the best known, are obtained with $N$ set at 100 .

Murtagh and Niwattisyawong (1982) propose a heuristic that uses MINOS, a large-scale nonlinear programming package, to solve simultaneously for both the locations and allocations. As the iterations proceed, the algorithm fixes any allocation decision variables $\left(w_{i j}\right)$ that reach either a value of zero or $w_{i}$, and then updates only the free variables. The update uses a quasi-Newton approximation of the Hessian matrix within the space of the free variables, and at nondifferentiable points, a subgradient suggested by Kuhn (1973).

Moreno et al. (1990) construct a "drop" heuristic that begins with an initial solution of $N$ clusters, where $N$ is chosen between $m$ and $2 m$. Then surplus facilities are dropped in a greedy manner until exactly $m$ are left. This method was tested on problem sizes of up to 900 customers and 10 facilities and obtained results comparable to the Cooper algorithm.

More recently, Bongartz et al. (1994) developed a projection method for solving the multisource Weber problem. Instead of assuming Euclidean distances, as is typically the case, the authors consider the more general $l_{p}$ norm. As in Murtagh and Niwattisyawong (1982), the new method solves simultaneously for location and allocation decision variables. Simple projection formulas on subspaces of the domain are derived (instead of solving the system of equations in general) and used to find descent directions. The algorithm is guaranteed to converge to a local minimum. The authors test a multistart version of their algorithm, where the initial solutions may be generated randomly or by partitioning customers in successive sets along a traveling salesman tour. The solutions are compared with multistart versions of Cooper's algorithm, Murtagh and Niwattisyawong (1982), and Chen (1983). The projection method generally outperforms the other heuristics, but in several of the reported test problems Cooper's algorithm comes in a close second. Thus, for the purposes of our current study, both of these methods will be considered as the state of the art.

Other heuristics have appeared after the projection method by Bongartz et al. (1994). These will for reference purposes be termed recent heuristics. Mladenović and Brimberg (1995) test a hybrid algorithm that takes random points in a $k$-exchange neighbourhood of the type used in the H-heuristics of Love and Juel (1982), and then applies Cooper's algorithm at each of these points. In Brimberg and Mladenović (1996a), elementary tabu search rules are added to the $\mathrm{H} 3$ heuristic to allow ascent moves away from a local optimum. Hansen et al. (1996) obtain an approximate solution by solving a related $p$-Median problem followed by the solution of single facility Weber problems. This idea was first suggested by Cooper (1963). A variable neighbourhood concept, introduced by Brimberg and Mladenović (1996b), systematically increases the number of exchanges $(k)$ of the H-type neighbourhood to expand the search radius about a local optimum. Variable neighbourhood search may be viewed as a new metaheuristic, with a wide range of possible applications in combinatorial optimization (see Mladenović 1995, Mladenović and Hansen 1997, and Hansen and Mladenović 1997).

There is a clear need for a comparative study of the heuristics that have appeared after the projection method of Bongartz et al. (1994). At the moment, there are several disconnected pieces, but no unified framework defining the current state of the art. Thus we begin the next section with a review of the recent heuristics. In addition, we present a new genetic algorithm and new facility relocation heuristics that we have developed. The defined relocation neighbourhood structures are used to conduct a simple local search —or alternatively, Tabu and variable neighbourhood searchesproducing several new methods. The subsequent section reports on an extensive empirical study comparing old, recent, and new heuristics. The last section summarizes our conclusions and suggests future directions of research.

In overview, the main objectives of our study are:

1. To update the state of the art by reviewing under one roof the several "recent" heuristics appearing after the projection method (Bongartz et al. 1994).

2. To add to this list "new" heuristics, and hybrid versions thereof, that we are currently studying.

3. To conduct an extensive empirical study comparing the new and recent heuristics together and with the old establishment (Bongartz et al. 1994 and Cooper 1963, 1964, 1967). Standard test problems will be used, but we will also consider much larger problem instances than previously reported in the literature. This will permit us to evaluate trends in performances of the various heuristics as problem size increases.

\section{RECENT HEURISTICS}

In this section we review several heuristic approaches to solve the multisource Weber problem, which have been recently developed by us. 


\subsection{Tabu Search (TS)}

This method (Brimberg and Mladenović 1996a) is an adaptation of the H3 heuristic of Love and Juel (1982) within the Tabu search framework (Glover 1989, 1990; Hansen and Jaumard 1990). A neighbourhood is constructed around a given (current) solution by considering all points obtained by a single exchange of a customer allocation from its current facility to another one. However, unlike the $\mathrm{H} 3$ heuristic, the tabu search algorithm will allow ascent moves from a local minimum. The parameters required by the basic method are ntabu and nbmax for the length of the tabu list and the number of moves allowed without an improvement in the objective function, respectively. It is understood below that the facilities are always optimally located with respect to the specified allocations of customers by solving up to $m$ independent single facility minisum problems.

Step 1 \{initialization\}. Obtain an initial solution by randomly partitioning the customer set $\{1, \ldots, n\}$ into $m$ mutually exclusive subsets $A_{j}$, and allocating $A_{j}$ to facility $j, \forall j=1, \ldots, m$ (located by using, e.g., the Weiszfeld procedure). Denote this solution by $X_{c}$, and let $Z_{c}$ equal the value of the objective function at $X_{c}$. Set $k=0$, $\left(X_{\text {best }}, Z_{\text {best }}\right)=\left(X_{c}, Z_{c}\right)$, and the tabu list $=\emptyset$.

Step 2 \{neighbourhood search\}. Consider all points $X_{i}$, $i=1, \ldots, n(m-1)$, in the one-exchange neighbourhood of $X_{c}$, except those that are not permitted by the tabu list. Retain the best solution $\left(X^{*}, Z^{*}\right)$ from among the neighbourhood points. If $Z^{*}<Z_{\text {best }}$, set $\left(X_{\text {best }}, Z_{\text {best }}\right)=\left(X^{*}, Z^{*}\right)$.

Step 3 move to adjacent point $\}$. Place the reverse exchange $\left(X^{*} \rightarrow X_{c}\right)$ at the bottom of the tabu list, and remove the top element in the list (using a FIFO rule) if the length exceeds $n t a b u$. If $Z^{*}<Z_{c}$ (descent move), set $k=0$; else, $k=k+1$. If $k>$ nbmax , STOP; else $\left(X_{c}, Z_{c}\right)=\left(X^{*}, Z^{*}\right)$ and return to Step 2.

\section{2. $p$-Median Heuristic (PM)}

The $p$-Median problem is a discrete version of the multisource Weber problem, where $p$ facility locations $(p=m)$ are to be chosen from $n$ nodes on a network representing the customer set. (For a review of the $p$-Median problem, see Mirchandani and Francis 1990.) The proposed $p$-Median heuristic (Hansen et al. 1996) solves the discrete problem optimally, where the facility locations are now restricted to the set of fixed points $\left\{a_{1}, \ldots, a_{n}\right\}$. It should be noted that the optimal solution of the continuous problem often has facilities located at or near customer sites. The travel distances between nodes are calculated with the Euclidean norm. The resulting $p$-Median problem is solved using the efficient code of Hanjoul and Peeters (1985).

Step 1. Define and solve a $p$-Median problem $(p=m)$ with the same customers and demands as in the continuous problem, and the set of fixed points $\left\{a_{1}, \ldots, a_{n}\right\}$ as the set of sites for locating facilities. Let $A_{j}$ be the subset of customers allocated to facility $j$ in the optimal solution, $j=1, \ldots, m$.
(Note that the $A_{j}$ are nonempty, mutually exclusive sets, and $\left.\bigcup_{j=1}^{m} A_{j}=\{1, \ldots, n\}.\right)$

Step 2. Solve $m$ independent continuous single facility minisum problems (e.g., using the Weiszfeld procedure), where facility $j$ serves exclusively subset $A_{j}, j=1, \ldots, m$. Let $x_{j}^{*}$ denote the optimal facility site thus obtained, $j=1, \ldots, m$.

Step 3. A heuristic solution for the multisource Weber problem is given by $\left\{\left(x_{j}^{*}, A_{j}\right) ; j=1, \ldots, m\right\}$, with objective function value

$Z_{P M}=\sum_{j=1}^{m} \sum_{i \in A_{j}} w_{i}\left\|x_{j}^{*}-a_{i}\right\|$.

A useful feature of the PM heuristic is that no parameters need to be specified by the analyst.

\subsection{Variable Neighbourhood Search (VNS)}

The variable neighbourhood search combines the elements of random search with a systematic way of exploring different regions of the solution space (Mladenovic 1995, Brimberg and Mladenović 1996b, Mladenović and Hansen 1997, Hansen and Mladenović 1997). If a given neighbourhood does not produce a better solution, we augment the neighbourhood in order to move further away from the current solution and resume the search. The neighbourhood structure used here is a generalization of the fixed neighbourhood used in the H-heuristics of Love and Juel (1982) and in the hybrid algorithm of Mladenović and Brimberg (1995). We define the $k$-neighbourhood of a given solution as the set of all possible surrounding points obtained by exactly $k$ exchanges of customer allocations from current facilities to new ones. This may be viewed as exchanging $k$ existing branches on a bipartite graph representation with $k$ new ones. The total number of points in the $k^{\text {th }}$ neighbourhood is bounded by

$\left(\begin{array}{l}n \\ k\end{array}\right)(m-1)^{k}$,

which increases exponentially with $k$. The procedure randomly chooses a specified number $b$ of points in this neighbourhood from which to conduct a local search with Cooper's algorithm. A basic form of VNS is outlined below. Generalizations of the method are discussed in Brimberg and Mladenović (1996b).

Step 1 \{initialization\}. Specify an initial solution, and run Cooper's algorithm to obtain a local optimum $\left(X_{c}\right)$. Set $k=1$.

Step 2 \{neighbourhood search\}.

(a) Select $b$ points at random in the $k$-neighbourhood of $X_{c}$.

(b) For each of these points run Cooper's algorithm to obtain local minima $X_{i}, i=1, \ldots, b$ (note that these solutions will not in general be all unique).

(c) Retain the best solution $X^{*} \in\left\{X_{i}, i=1, \ldots, b\right\}$. 
(d) If $X^{*}$ is a better solution than $X_{c}, X_{c}=X^{*}, k=1$, and return to the beginning of Step 2; otherwise, proceed to the next step.

Step 3 \{augmenting the neighbourhood\}.

(a) $k=k+1$.

(b) If $k \leqslant k_{\max }$, return to Step 2 ; otherwise, if the stopping criterion is not satisfied, set $k=1$ and return to Step 2; else STOP (final solution is $X_{c}$ ).

The parameters to be specified in VNS are $b$ and $k_{\max }$. In the heuristic by Mladenović and Brimberg (1995), the neighbourhood size is fixed at a specified parameter value $(k)$. Random points are chosen in the neighbourhood, and the local descent from each of these points is carried out using Cooper's algorithm as above. This procedure referred to as fixed neighbourhood search, as well as VNS, may be easily modified to allow ascent moves.

\section{NEW HEURISTICS}

In this section we describe a new genetic algorithm and a framework for new facility relocation heuristics.

\subsection{Genetic Algorithm (GA)}

Unlike random search methods that do not use any previous information, the genetic algorithm attempts to construct improved solutions from predecessors in an evolutionary type process (Holland 1975). In this respect the genetic algorithm may be thought of as a more intelligent stochastic search technique. A genetic algorithm has already been developed for the multisource Weber problem by Houck et al. (1996). We give below the general framework of the algorithm followed by details of our implementation.

Step 1. Generate $N$ different initial solutions (the population of solutions).

Step 2. Sort the population in nonincreasing order of solution quality measured by the value of the objective function.

Step 3.

Repeat:

(a) Select two solutions from the population,

(b) Mix these solutions with a cross-over operator to create a new solution,

(c) Modify the new solution with a mutation operator,

(d) Insert the modified solution in the population and sort,

(e) Remove solutions from the population with a culling operator,

until a stopping criterion is satisfied.

To determine an initial solution in Step 1 of GA, the facilities are located at $m$ randomly chosen fixed points. Using these starting locations, the alternating algorithm of Cooper is applied until a local minimum is reached. The process is repeated until $N$ local minima are obtained to make up the population.
The selection operator in Step 3(a) generates two instances $y_{1}, y_{2}$ of a random variable uniformly distributed in the interval $(0,1)$. The first solution selected from the sequenced population is identified as $s_{1}=\left\lfloor y_{1}^{2} \cdot Q+1\right\rfloor$, where $Q$ is the number of solutions currently in the population and $\lfloor y\rfloor$ denotes the largest integer value less than or equal to $y$. The second solution is given by

$s_{2}= \begin{cases}s_{2}^{\prime}, & \text { if } s_{2}^{\prime}<s_{1}, \\ s_{2}^{\prime}+1, & \text { otherwise, }\end{cases}$

where $s_{2}^{\prime}=\left\lfloor y_{2}^{2} \cdot(Q-1)+1\right\rfloor$. Note that the squaring of $y_{1}$ and $y_{2}$ in the above formulas tends to generate smaller integer values for $s_{1}$ and $s_{2}$; that is, the tendency is to select the best solutions from the population in line with a survivalof-the-fittest strategy.

The cross-over operation combines the features for the two existing solutions, $s_{1}$ and $s_{2}$ (the parents), to produce a new solution $s_{3}$ (the child). In our implementation, each facility $j$ is added to $s_{3}$ at a site it occupies in $s_{1}$ or $s_{2}$. A minimal separation distance $d_{\min }$ between facilities is specified to spread them out among the customers and avoid duplication of good sites. We use a $d_{\min }$ equal to the smallest distance between two customers. Let $x_{i j}$ be the site of facility $j$ in solution $i$. The cross-over operation works as follows. First set $x_{31}=x_{11}$ or $x_{21}$, with equal probability. Then, for each $j=2, \ldots, m$, calculate with distance function $d(.,$.$) :$

$d_{1}=\min _{t<j} d\left(x_{3 t}, x_{1 j}\right), \quad d_{2}=\min _{t<j} d\left(x_{3 t}, x_{2 j}\right)$.

If $\left(d_{1}<d_{\text {min }}\right)$ and $\left(d_{2}>d_{\text {min }}\right)$, set $x_{3 j}=x_{2 j}$; else if $\left(d_{2}<d_{\text {min }}\right)$ and $\left(d_{1}>d_{\min }\right)$, set $x_{3 j}=x_{1 j}$; else set $x_{3 j}=x_{1 j}$ or $x_{2 j}$ with equal probability.

The mutation operator in Step 3(c) is simply a local improvement on the new solution $s_{3}$ using the Cooper algorithm to obtain a local minimum. If the population size exceeds a specified limit $Q_{\max }$, the culling operator removes the worst solution in Step 3(e). The process will continue producing new generations of solutions indefinitely if it is not stopped. The stopping criterion is typically a limit on the number of iterations or on the execution time or the convergence of the algorithm is detected (i.e., all solutions of the population are the same). Thus, in summary, we need to specify the parameters $N, Q_{\max }, d_{\min }$, and a stopping criterion to implement the procedure.

\subsection{Relocation Heuristics}

Until now, local searches have been conducted in a neighbourhood of the current solution defined by a fixed number of customer-to-facility reallocations. We propose here a new local search procedure that constructs its neighbourhood as the set of points obtained by a given number of facility relocations. The simplest construction considers the relocation of a single facility to any unoccupied customer location (i.e., a customer that does not have a facility coincident with it). This one-exchange neighbourhood has been used before in network location problems; e.g., see Teitz and Bart (1968) 
for the $p$-Median problem. Because there are $m$ candidates to choose from, and as many as $n$ customer sites at which to reposition them, there are $O(m n)$ points in the resulting neighbourhood.

Local searches that visit all the points in the neighbourhood will be referred to as interchange $(\mathrm{CH})$ heuristics. Various strategies may be employed in this context to trade off the accuracy or depth of the search at a neighbourhood point with speed. For example, the facilities may always be optimally located in continuous space (for the specified allocations), or forced to remain only at customer sites. In the latter case, the heuristic is solving the related discrete $m$-Median problem. The algorithm would adjust the facility locations in continuous space at well-defined times. The net effect would be to allow more iterations (with less precision) in the same amount of CPU time.

Instead of visiting all points in the interchange neighbourhood, an alternative strategy referred to as drop and add (DA) could be used. This procedure has been applied with success in other settings such as the Traveling Salesman Problem (see for example Gendreau et al. 1992) and the p-Median problem (e.g., Rolland et al. 1996); however, to the best of our knowledge, this is the first application in continuous location-allocation problems. The DA method decides, using some criterion, which is the best facility to drop, and only then, by another criterion, where is the best site to reinsert it. It follows that $O(m+n)$ points are investigated in place of $O(\mathrm{mn})$ of the interchange procedure. However, visiting $O(m n)$ solutions in an interchange neighbourhood may not be more time consuming than visiting $O(m+n)$ solutions in a DA neighbourhood because of the following facts: (1) A good drop strategy may be time consuming (if, for example, a local minimum is obtained each time for the remaining $m-1$ facilities) and likewise for the add move; (2) As shown by Whitaker (1983), the points in an interchange neighbourhood may be updated efficiently in a $p$-Median problem. We found that these results could also be applied to the continuous relocation neighbourhood we constructed.

A basic form of the DA heuristic follows.

Step 1 \{initialization\}. Find an initial solution $X_{c}$, and let $Z_{c}$ be the corresponding value of the objective function.

Step $2\{$ drop $\}$. Delete a facility at site $\left(x_{j_{d e l} 1}, x_{j_{d e l}}\right)$ using some criterion. (All other facilities remain in their current locations.)

Step 3 \{add $\}$. Reinsert the facility at an unoccupied customer location $\left(a_{i_{\text {new }}}, a_{i_{\text {new }}} 2\right)$ according to some other criterion.

Step 4 \{local improvement $\}$. Use Cooper's algorithm and the modified set of facility locations to find a local minimum $\left(X^{*}, Z^{*}\right)$. If $Z^{*}<Z_{c}$, save the new currently best solution, $\left(X_{c}, Z_{c}\right)=\left(X^{*}, Z^{*}\right)$, and return to Step 2; otherwise STOP.

The key features in the DA heuristic are seen to be the criteria used in Steps 2 and 3. Several deletion and insertion rules were investigated, but for brevity we will report only on the more successful ones. The three best criteria for dropping a facility were found to be:
Brimberg, Hansen, Mladenović, and Taillard

1. Drop least useful $(D L U)$. Here each facility $j$ is deleted in turn, and a local minimum $W_{j}$ is obtained by Cooper's algorithm for the remaining $(m-1)$ facilities. The facility to be dropped corresponds to the minimum-valued $W_{j}$, i.e., $W_{j_{d e l}}=\min \left\{W_{j}, j=1, \ldots, m\right\}$.

2. Drop by second closest criterion (DSC). Find the second closest facility to each customer. Now temporarily remove a facility $j$. Let $r_{j}$ be its contribution in the current objective function, and let $s_{j}$ equal the weighted sum of distances between customers temporarily without a facility and their second closest facility. Repeat the preceding step for $j=1, \ldots, m$. Then facility $j_{d e l}$ corresponds to the minimum difference, $s_{j}-r_{j}$.

3. Drop by minimum potential criterion $(D M P)$. Define the potential of each facility $j$ as the product $r_{j} d_{j}$, where $r_{j}$ is its contribution to the objective function and $d_{j}$ the distance to its closest facility. If facility $j$ coincides with a customer $i$, then set $r_{j}=r_{j}+w_{i}$. Drop the facility $j_{d e l}$ with the minimum potential.

The first drop procedure is intuitively the most appealing because it identifies a facility whose removal will cause the least increase in the objective function. However, Cooper's algorithm must be called $m$ times in each iteration, and hence this procedure becomes time consuming for larger problem instances. The second and third drop procedures, on the other hand, are much faster because a local improvement of the solution for the remaining $(m-1)$ facilities is not carried out.

Analogous versions of the drop step may be constructed for the add step. We mention only two of these:

1. Add most useful (AMU). Insert the deleted facility at an unoccupied customer location. Find a local minimum using Cooper's algorithm and the new set of facility sites. Repeat for each unoccupied customer location and retain the best solution.

2. Add by second closest criterion (ASC). Insert the deleted facility at an unoccupied customer location $i$. Reallocate those customers who are now closer to the newly inserted facility than to their current facility (which becomes the second closest). Calculate the decrease in the objective function $\left(s_{i}-r_{i}\right)$ attributed to the given insertion point. Repeat for each unoccupied customer location, and retain the insertion point which maximizes $\left(s_{i}-r_{i}\right)$.

The interchange and drop/add neighbourhoods may be enlarged by increasing the number of facility relocations from the current solution. In addition, we may allow ascent moves in the defined neighbourhood. This gives rise to a host of new heuristics based on tabu search and variable neighbourhood search. These algorithms apply the same steps as before, except that the reallocation neighbourhoods are now replaced by the newly defined relocation neighbourhoods.

\section{COMPUTATIONAL RESULTS}

An extensive empirical study was carried out to compare the various heuristics - old, recent, and new-in a unified setting. We considered the following four problem 
Table 1. Optimal and best known values for test problems.

\begin{tabular}{|c|c|c|c|c|c|c|c|}
\hline & $n=50$ & & $n=287$ & & $n=654$ & & $n=1060$ \\
\hline$m$ & $\begin{array}{l}\text { Optimal } \\
\text { Value }\end{array}$ & $m$ & $\begin{array}{l}\text { Optimal } \\
\text { Value }\end{array}$ & $m$ & $\begin{array}{c}\text { Best Known } \\
\text { Value }\end{array}$ & $m$ & $\begin{array}{c}\text { Best Known } \\
\text { Value }\end{array}$ \\
\hline 2 & 135.5222 & 2 & 14427.5930 & 2 & 815313.2961 & 5 & 1851879.9 \\
\hline 3 & 105.2139 & 3 & 12095.4422 & 3 & 551062.8811 & 10 & 1249564.8 \\
\hline 4 & 84.1536 & 4 & 10661.4766 & 4 & 288190.9860 & 15 & 980132.1 \\
\hline 5 & 72.2369 & 5 & 9715.6275 & 5 & 209068.7935 & 20 & 828802.0 \\
\hline 6 & 60.9713 & 6 & 8787.5568 & 6 & 180488.2126 & 25 & 722061.2 \\
\hline 7 & 54.5020 & 7 & 8160.3230 & 7 & 163704.1681 & 30 & 638263.0 \\
\hline 8 & 49.9393 & 8 & 7564.2949 & 8 & 147050.7904 & 35 & 577526.6 \\
\hline 9 & 45.6884 & 9 & 7088.1283 & 9 & 130936.1241 & 40 & 529866.2 \\
\hline 10 & 41.6851 & 10 & 6705.0356 & 10 & 115339.0328 & 45 & 489650.0 \\
\hline 11 & 38.0205 & 11 & 6351.5910 & 11 & 100133.2007 & 50 & 453164.0 \\
\hline 12 & 35.0551 & 12 & 6033.0474 & 12 & 94152.0550 & 55 & 422770.0 \\
\hline 13 & 32.3067 & 13 & 5725.1853 & 13 & 89454.7613 & 60 & 397784.4 \\
\hline 14 & 29.6559 & 14 & 5469.6478 & 14 & 84807.6690 & 65 & 376759.5 \\
\hline 15 & 27.6282 & 15 & 5224.7028 & 15 & 80177.0422 & 70 & 357385.0 \\
\hline 16 & 25.7427 & 16 & 4981.9608 & 20 & 63389.0238 & 75 & 340242.0 \\
\hline 17 & 23.9900 & 17 & 4755.1890 & 25 & 52209.5106 & 80 & 326053.2 \\
\hline 18 & 22.2851 & 18 & 4547.3651 & 30 & 44705.1921 & 85 & 313738.2 \\
\hline 19 & 20.6399 & 19 & 4342.0648 & 35 & 39257.2685 & 90 & 302837.0 \\
\hline 20 & 19.3560 & 20 & 4148.8443 & 40 & 35704.4076 & 95 & 292875.1 \\
\hline 21 & 18.0826 & 25 & 3348.7101 & 45 & 32306.9721 & 100 & 283113.0 \\
\hline 22 & 16.8220 & 30 & 2716.9071 & 50 & 29338.0106 & 105 & 274576.0 \\
\hline 23 & 15.6136 & 35 & 2238.1839 & 55 & 26699.1699 & 110 & 265801.0 \\
\hline 24 & 14.4431 & 40 & 1900.8361 & 60 & 24504.3952 & 115 & 257605.0 \\
\hline 25 & 13.3016 & 45 & 1630.3115 & 65 & 22747.0996 & 120 & 249584.0 \\
\hline 26 & 12.3016 & 50 & 1402.5836 & 70 & 21468.1543 & 125 & 242930.0 \\
\hline 27 & 11.4193 & 55 & 1203.9849 & 75 & 20312.9668 & 130 & 236154.0 \\
\hline 28 & 10.4759 & 60 & 1055.1391 & 80 & 19193.8848 & 135 & 230431.0 \\
\hline 29 & 9.5936 & 65 & 924.5547 & 85 & 18316.5391 & 140 & 224504.0 \\
\hline 30 & 8.7963 & 70 & 814.2238 & 90 & 17544.3516 & 145 & 218279.0 \\
\hline 31 & 7.9666 & 75 & 730.0434 & 95 & 16786.3887 & 150 & 212926.0 \\
\hline 32 & 7.1814 & 80 & 655.3788 & 100 & 16087.6846 & & \\
\hline 33 & 6.4567 & 85 & 588.3680 & 105 & 15436.4004 & & \\
\hline 34 & 5.7484 & 90 & 529.2126 & 110 & 14830.1602 & & \\
\hline 35 & 5.0483 & 95 & 480.8592 & 115 & 14381.0566 & & \\
\hline 36 & 4.5471 & 100 & 441.2417 & 120 & 13921.5498 & & \\
\hline
\end{tabular}

configurations: the well-known 50-customer problem in Eilon et al. (1971), the 287-customer ambulance problem from Bongartz et al. (1994), and a 654- and 1060-customer problem listed in the TSP library (Reinelt 1991). The weights $\left(w_{i}\right)$ were taken directly from Bongartz et al. for the 287customer problem; otherwise, unit weights were specified throughout. In each case, the number of facilities to locate was varied over a wide range. This provided a large number of problem instances from comparatively small sizes to much larger instances than previously reported in the literature. Thus we were able to investigate the performance of the heuristics over an extensive range of problem difficulty.

The different methods were compared on the basis of equivalent CPU times. Each problem instance was solved initially by 100 runs of Cooper's alternating algorithm from randomly generated starting points. (The multistart version of Cooper's algorithm will be referred to as MALT from this point on.) The resulting CPU time was then used as a stopping criterion for the other heuristics. That is, the algorithm would be terminated at the completion of a local search if the total elapsed CPU time exceeded the stopping criterion; otherwise the iterations were allowed to continue. In cases where the stopping criterion could not be applied, such as the $p$-Median algorithm, actual CPU times to complete the first solution are reported.

All methods were programmed in FORTRAN 77 except GA, which used $\mathrm{C}++$. The codes were compiled using an optimizing option $(g++-O 3$ for $\mathrm{C}++$, and $f 77-c g 92-O 4$ for FORTRAN) and run on a SUN SPARC station 10.

\section{Old and Recent Heuristics}

Results for the "old" and "recent" heuristics are reported in Tables 2 to 5. Where applicable, two sets of values are given: the average and the best value of the objective function found from 10 separate runs of the algorithm. These results are expressed as a percent deviation from the best known solutions (listed in Table 1). It should be noted that the best known solutions listed for the 50- and 287-customer 
Table 2. Old/recent heuristics and the 50-customer problem.

\begin{tabular}{rrrrrrrrrrrrr}
\hline & \multirow{2}{*}{ CPU } & \multicolumn{2}{c}{ MALT } & \multicolumn{2}{c}{ TS } & \multicolumn{2}{c}{ VNS-1 } & \multicolumn{2}{c}{ PROJ } & \multicolumn{2}{c}{ PM-1 } \\
$m$ & Time & Av. & Best & Av. & Best & Av. & Best & Av. & Best & CPU & Best \\
\hline 2 & 0.94 & 0.00 & 0.00 & 3.71 & 0.00 & 3.39 & 0.00 & 1.45 & 0.00 & 0.06 & 0.05 \\
3 & 1.59 & 0.00 & 0.00 & 1.51 & 0.00 & 1.35 & 0.00 & 2.24 & 0.00 & 0.06 & 0.26 \\
4 & 2.21 & 0.00 & 0.00 & 3.04 & 0.00 & 1.23 & 0.00 & 4.26 & 0.00 & 0.06 & 0.02 \\
5 & 2.82 & 0.00 & 0.00 & 1.09 & 0.00 & 0.15 & 0.00 & 2.93 & 0.00 & 0.08 & 0.63 \\
6 & 3.75 & 0.00 & 0.00 & 6.41 & 0.00 & 1.25 & 0.00 & 2.65 & 0.00 & 0.05 & 0.21 \\
7 & 4.00 & 0.00 & 0.00 & 3.51 & 0.00 & 2.97 & 0.00 & 4.53 & 0.25 & 0.05 & 0.24 \\
8 & 4.23 & 0.06 & 0.00 & 4.61 & 0.00 & 0.63 & 0.00 & 4.78 & 0.72 & 0.05 & 0.00 \\
9 & 4.26 & 0.38 & 0.00 & 4.00 & 0.79 & 1.78 & 0.00 & 6.19 & 0.91 & 0.05 & 0.00 \\
10 & 4.27 & 0.51 & 0.00 & 8.16 & 1.77 & 2.65 & 0.00 & 8.14 & 2.40 & 0.05 & 0.00 \\
11 & 4.31 & 2.24 & 1.34 & 11.73 & 5.83 & 2.53 & 0.00 & 9.44 & 1.57 & 0.05 & 0.00 \\
12 & 4.27 & 2.65 & 0.35 & 13.75 & 7.84 & 1.06 & 0.00 & 9.44 & 0.61 & 0.05 & 0.00 \\
13 & 4.24 & 3.90 & 1.57 & 15.51 & 2.22 & 1.45 & 0.00 & 10.40 & 3.33 & 0.05 & 0.30 \\
14 & 4.18 & 4.22 & 2.18 & 16.49 & 6.35 & 1.91 & 0.00 & 12.48 & 5.65 & 0.05 & 0.00 \\
15 & 4.14 & 4.73 & 0.84 & 19.55 & 9.06 & 1.39 & 0.00 & 11.56 & 5.33 & 0.05 & 0.41 \\
16 & 4.05 & 4.93 & 1.53 & 16.88 & 8.98 & 0.76 & 0.00 & 11.62 & 5.44 & 0.06 & 0.00 \\
17 & 3.91 & 4.88 & 1.11 & 20.88 & 9.62 & 0.03 & 0.00 & 12.78 & 5.75 & 0.05 & 0.00 \\
18 & 3.90 & 6.39 & 3.70 & 20.72 & 8.73 & 0.32 & 0.01 & 14.70 & 2.97 & 0.06 & 0.00 \\
19 & 3.80 & 5.66 & 1.41 & 21.11 & 10.15 & 0.04 & 0.00 & 15.01 & 2.91 & 0.05 & 0.00 \\
20 & 3.75 & 5.81 & 0.68 & 19.87 & 6.34 & 0.20 & 0.00 & 13.37 & 3.63 & 0.05 & 0.04 \\
21 & 3.67 & 5.77 & 2.11 & 13.45 & 6.40 & 0.16 & 0.00 & 12.60 & 1.34 & 0.09 & 0.35 \\
22 & 3.60 & 6.04 & 3.76 & 16.17 & 5.18 & 0.30 & 0.00 & 13.57 & 5.34 & 0.05 & 0.44 \\
23 & 3.52 & 4.93 & 1.00 & 23.38 & 11.74 & 0.42 & 0.00 & 14.42 & 4.34 & 0.09 & 0.89 \\
24 & 3.46 & 5.77 & 2.92 & 22.24 & 9.64 & 0.27 & 0.00 & 13.91 & 0.37 & 0.10 & 0.95 \\
25 & 3.38 & 5.58 & 2.50 & 23.68 & 9.64 & 0.19 & 0.00 & 13.60 & 2.00 & 0.05 & 0.82 \\
\hline \multirow{2}{*}{ Av. } & 3.59 & 3.10 & 1.12 & 12.98 & 5.01 & 1.10 & 0.00 & 9.42 & 2.29 & 0.06 & 0.23 \\
\hline & & & & & & & & & & &
\end{tabular}

problems are also known to be global optimal solutions (Krau 1997). The cPu times (in seconds) listed in the tables are for 10 runs of MALT (with 100 restarts of Cooper's algorithm in each run), thus giving the total time to obtain the best solution.

We note that the projection method (PROJ) of Bongartz et al. (1994) uses the original code supplied by the authors. This code permits the choice between random or travelling salesman solutions as starting points. The results reported here for PROJ apply to the type of starting point that gave the best average performance over all problem instances in the table (random initial solutions in Table 3, and travelling salesman solutions for Tables 2, 4, and 5). Also note that the heuristics are all augmented where applicable by an insertion procedure to eliminate degenerate local minima that occur when one or more facilities are at locations that do not serve any customers (see Brimberg and Mladenović 1998).

Some general observations are inferred from a comparison of the results in Tables 2 to 5. These are summarized below.

ObSERVATion 1. At the lower values of $m$ in each table, the old heuristics (MALT and the projection method (PROJ) of Bongartz et al.) perform as well as or better than the recent heuristics. This implies that random restarts are an effective solution strategy for "smaller" problem instances. This relates to the existence of relatively few local minima.
ObSERVATION 2. The relatively poor performance of MALT and PROJ for higher values of $m$ may be attributed to the fact that the number of local minima increases with problem size at an exponential rate, giving rise to a central-limit catastrophe (Boese et al. 1994). As a result, procedures that use random restarts lose their effectiveness. Also note that PROJ takes up to $80 \%$ longer than MALT to find a local minimum. Nonetheless, PROJ gives the best performance overall in Table 5 for the 1060 -customer problem. This may be because of the use of travelling salesman solutions in PROJ to generate starting points.

ObSeRVATIOn 3. The Tabu search method (TS-1) performs poorly in comparison to MALT for large problem sizes (see Table 2). This is attributed to the neighbourhood structure in TS, which results in a very slow local descent or ascent. Thus, relatively few iterations are completed within the imposed time limit. For this reason, we report TS-1 only in Table 2. For small problem sizes, TS- 1 is seen to be competitive with the other methods within the imposed time limit.

OBSERVATION 4. The variable neighbourhood search (VSN1) reported in Tables 2 to 5 uses in all cases parameter values of $b=1$ (number of points randomly selected in a given neighbourhood), and $k_{\max }=n$ (maximum number of customer reallocations or largest neighbourhood). No attempt was made to find the best parameter values for individual problems, but rather, a "parameterless" version 
Table 3. Old/recent heuristics and the 287-customer problem.

\begin{tabular}{|c|c|c|c|c|c|c|c|c|c|c|c|}
\hline \multirow[b]{2}{*}{$m$} & \multirow{2}{*}{$\begin{array}{l}\text { CPU } \\
\text { Time }\end{array}$} & \multicolumn{2}{|c|}{ MALT } & \multicolumn{2}{|c|}{ FNS } & \multicolumn{2}{|c|}{ VNS-1 } & \multicolumn{2}{|c|}{ PROJ } & \multicolumn{2}{|c|}{ PM-1 } \\
\hline & & Av. & Best & Av. & Best & Av. & Best & Av. & Best & $\mathrm{CPU}$ & Best \\
\hline 2 & 10.34 & 0.04 & 0.00 & 0.00 & 0.00 & 0.00 & 0.00 & 4.22 & 0.00 & 2.28 & 0.42 \\
\hline 3 & 18.63 & 0.00 & 0.00 & 0.00 & 0.00 & 0.00 & 0.00 & 10.12 & 0.00 & 2.04 & 0.04 \\
\hline 4 & 20.04 & 0.01 & 0.01 & 4.12 & 0.01 & 3.67 & 0.01 & 12.27 & 0.00 & 2.14 & 0.01 \\
\hline 5 & 23.24 & 0.89 & 0.21 & 2.93 & 0.20 & 4.29 & 0.20 & 16.47 & 0.00 & 2.26 & 0.01 \\
\hline 6 & 25.94 & 3.39 & 3.21 & 2.89 & 0.03 & 3.31 & 3.21 & 18.57 & 3.49 & 1.60 & 0.03 \\
\hline 7 & 27.48 & 3.81 & 0.79 & 1.35 & 0.39 & 3.06 & 0.03 & 22.47 & 4.65 & 2.22 & 0.06 \\
\hline 8 & 29.53 & 4.22 & 2.36 & 1.50 & 0.01 & 2.07 & 0.01 & 26.84 & 3.07 & 2.15 & 0.56 \\
\hline 9 & 30.60 & 3.10 & 0.54 & 1.15 & 0.00 & 2.02 & 0.00 & 21.39 & 5.74 & 2.09 & 0.58 \\
\hline 10 & 32.21 & 3.05 & 0.96 & 0.39 & 0.30 & 0.89 & 0.32 & 26.21 & 8.09 & 2.63 & 0.72 \\
\hline 11 & 33.49 & 2.08 & 0.97 & 0.65 & 0.12 & 0.60 & 0.00 & 22.76 & 9.28 & 2.27 & 0.59 \\
\hline 12 & 34.87 & 2.60 & 0.95 & 0.31 & 0.00 & 0.41 & 0.00 & 20.31 & 10.28 & 2.43 & 0.44 \\
\hline 13 & 35.63 & 2.77 & 2.01 & 0.39 & 0.00 & 0.26 & 0.00 & 29.88 & 12.00 & 2.05 & 0.69 \\
\hline 14 & 36.99 & 2.47 & 1.34 & 0.34 & 0.01 & 0.15 & 0.01 & 25.59 & 9.70 & 2.38 & 0.42 \\
\hline 15 & 37.17 & 2.09 & 1.57 & 0.24 & 0.01 & 0.58 & 0.01 & 27.96 & 11.20 & 2.39 & 0.16 \\
\hline 16 & 38.32 & 2.43 & 1.34 & 0.39 & 0.01 & 0.49 & 0.01 & 29.71 & 14.14 & 1.88 & 0.26 \\
\hline 17 & 38.67 & 2.51 & 1.08 & 0.49 & 0.01 & 1.06 & 0.01 & 27.72 & 16.60 & 1.92 & 0.15 \\
\hline 18 & 39.57 & 2.18 & 0.82 & 0.50 & 0.06 & 0.91 & 0.06 & 31.35 & 18.57 & 2.01 & 0.22 \\
\hline 19 & 39.72 & 3.07 & 1.66 & 0.99 & 0.01 & 0.95 & 0.40 & 35.82 & 15.28 & 1.81 & 0.23 \\
\hline 20 & 39.88 & 3.66 & 2.27 & 1.41 & 0.14 & 1.12 & 0.28 & 34.10 & 16.54 & 2.48 & 0.24 \\
\hline 25 & 42.83 & 5.81 & 3.40 & 2.32 & 0.62 & 2.85 & 0.87 & 46.74 & 25.05 & 2.31 & 0.02 \\
\hline 30 & 48.39 & 6.77 & 4.13 & 3.11 & 0.36 & 3.41 & 2.29 & 52.61 & 25.07 & 2.45 & 0.06 \\
\hline 35 & 53.64 & 8.05 & 4.80 & 3.63 & 0.01 & 2.73 & 0.05 & 50.82 & 25.26 & 2.39 & 0.02 \\
\hline 40 & 58.65 & 8.83 & 4.70 & 5.79 & 3.99 & 2.61 & 1.22 & 63.24 & 47.01 & 2.77 & 0.09 \\
\hline 45 & 63.28 & 10.58 & 7.44 & 5.63 & 4.29 & 3.21 & 1.43 & 49.31 & 33.76 & 2.20 & 0.14 \\
\hline 50 & 67.32 & 12.07 & 5.93 & 7.76 & 5.49 & 4.71 & 2.12 & 54.70 & 38.97 & 2.60 & 0.01 \\
\hline 55 & 70.06 & 15.77 & 12.58 & 8.09 & 3.68 & 6.14 & 2.77 & 53.05 & 28.92 & 2.53 & 0.02 \\
\hline 60 & 74.69 & 18.49 & 8.88 & 10.76 & 5.25 & 4.76 & 1.83 & 49.57 & 37.85 & 2.94 & 0.03 \\
\hline 65 & 77.99 & 21.63 & 17.14 & 11.63 & 6.39 & 6.95 & 4.21 & 45.28 & 29.04 & 4.25 & 0.17 \\
\hline 70 & 81.45 & 25.13 & 13.91 & 13.31 & 9.84 & 9.32 & 2.36 & 47.22 & 25.46 & 2.65 & 0.10 \\
\hline 75 & 83.79 & 27.28 & 21.81 & 12.26 & 5.08 & 7.61 & 2.69 & 37.50 & 22.72 & 2.75 & 0.18 \\
\hline 80 & 86.07 & 28.30 & 22.23 & 14.62 & 12.25 & 9.91 & 4.18 & 37.65 & 20.06 & 2.68 & 0.18 \\
\hline 85 & 88.24 & 33.95 & 27.12 & 15.68 & 11.12 & 10.50 & 5.59 & 34.29 & 22.21 & 20.04 & 0.13 \\
\hline 90 & 89.97 & 32.89 & 21.65 & 17.01 & 14.01 & 8.86 & 4.66 & 30.85 & 17.85 & 2.07 & 0.09 \\
\hline 95 & 91.66 & 37.43 & 28.31 & 16.52 & 12.77 & 9.48 & 5.01 & 31.45 & 16.98 & 2.26 & 0.10 \\
\hline 100 & 92.99 & 39.41 & 30.12 & 19.00 & 15.26 & 8.86 & 4.47 & 29.34 & 12.12 & 2.17 & 0.00 \\
\hline Av. & 50.38 & 10.76 & 7.32 & 5.35 & 3.19 & 3.65 & 1.44 & 33.07 & 16.77 & 2.86 & 0.20 \\
\hline
\end{tabular}

was implemented to see how the algorithm in its simplest form could perform over all problem sizes.

Referring to the average error summary in the tables, we observe that VNS-1 significantly outperformed MALT within the same CPU time. For the 50-customer problem, the best solution obtained by VNS-1 was optimal in almost all cases! However, the results were not uniform for the other problem sets. It is also interesting to note that the fixed neighbourhood search (FNS) with number of reallocations $k=n / 2$, obtained substantially better results than VNS-1 for the problem sets in Tables 4 and 5. This implies that the variable neighbourhood search is sensitive to the parameter settings. Thus, for best results, the parameters should be adjusted for individual problems (or CPU time increased). Alternatively, $b$ and $k_{\max }$ may vary according to an intensification/diversification strategy during the execution of the algorithm.

Observation 5. The solutions obtained by the $p$-Median algorithm (PM-1) are very good in comparison to the other methods reported in Tables 2 to 5 . With the exception of the 50 -customer problem, PM-1 outperforms VNS-1 by a considerable margin. However, the execution time for PM-1 to complete a solution far exceeded the time limit imposed on VNS-1, so a direct comparison of the two methods cannot be made. Note that results for PM-1 are not listed for execution times exceeding a 3000-second limit. (This is also the reason PM-1 is not included in Table 5.)

\section{New Descent Relocation Heuristics}

We begin with a discussion of results for the drop/add (DA) algorithm. As noted in the description of this method in $\S 3$, several criteria may be selected to determine which facility to remove and where to insert it back. This provides a large number of possible drop/add strategies. We will report on only a few of the more promising combinations.

Table 6 provides results on four DA strategies for the 287-customer problem. The Av. column gives the average result from 10 random restarts, while the next column lists 
Table 4. Old/recent heuristics and the 654-customer problem.

\begin{tabular}{|c|c|c|c|c|c|c|c|c|c|c|c|}
\hline \multirow[b]{2}{*}{$m$} & \multirow{2}{*}{$\begin{array}{l}\text { CPU } \\
\text { Time }\end{array}$} & \multicolumn{2}{|c|}{ MALT } & \multicolumn{2}{|c|}{ FNS } & \multicolumn{2}{|c|}{ VNS-1 } & \multicolumn{2}{|c|}{ PROJ } & \multicolumn{2}{|c|}{ PM-1 } \\
\hline & & Av. & Best & Av. & Best & Av. & Best & Av. & Best & $\mathrm{CPU}$ & Best \\
\hline 2 & 13.10 & 0.00 & 0.00 & 0.00 & 0.00 & 0.00 & 0.00 & 0.00 & 0.00 & 33.19 & 0.00 \\
\hline 3 & 26.29 & 0.00 & 0.00 & 0.16 & 0.00 & 0.14 & 0.00 & 0.00 & 0.00 & 37.67 & 0.20 \\
\hline 4 & 49.82 & 0.00 & 0.00 & 12.84 & 0.00 & 12.84 & 0.00 & 0.00 & 0.00 & 25.12 & 0.00 \\
\hline 5 & 49.99 & 0.00 & 0.00 & 0.00 & 0.00 & 0.00 & 0.00 & 16.71 & 0.00 & 33.39 & 0.00 \\
\hline 6 & 55.43 & 3.54 & 0.00 & 0.57 & 0.00 & 0.00 & 0.00 & 3.07 & 0.00 & 36.08 & 0.00 \\
\hline 7 & 60.96 & 0.60 & 0.08 & 0.52 & 0.00 & 0.52 & 0.00 & 2.44 & 0.00 & 31.06 & 0.00 \\
\hline 8 & 61.92 & 0.65 & 0.00 & 4.60 & 0.72 & 4.41 & 0.72 & 0.89 & 0.00 & 39.62 & 0.00 \\
\hline 9 & 61.25 & 0.40 & 0.40 & 9.33 & 0.40 & 9.40 & 0.40 & 0.78 & 0.40 & 35.69 & 0.00 \\
\hline 10 & 1.26 & 5.18 & 0.00 & 19.23 & 11.56 & 19.08 & 9.21 & 3.11 & 0.00 & 37.74 & 0.04 \\
\hline 11 & 60.30 & 14.87 & 9.03 & 15.76 & 0.00 & 24.60 & 20.92 & 8.08 & 0.00 & 32.86 & 0.07 \\
\hline 12 & 61.13 & 13.56 & 10.87 & 15.03 & 11.29 & 21.63 & 1.44 & 13.48 & 13.07 & 48.28 & 0.00 \\
\hline 13 & 61.73 & 15.30 & 14.19 & 9.82 & 0.57 & 19.13 & 0.57 & 8.99 & 1.60 & 129.70 & 0.01 \\
\hline 14 & 63.41 & 17.78 & 5.54 & 5.97 & 0.60 & 20.73 & 3.16 & 13.94 & 1.68 & 138.97 & 0.00 \\
\hline 15 & 64.86 & 19.77 & 6.68 & 6.18 & 0.58 & 16.76 & 3.33 & 12.25 & 1.75 & 98.07 & 0.01 \\
\hline 20 & 89.47 & 27.18 & 23.24 & 8.03 & 3.94 & 21.28 & 11.27 & 22.76 & 7.81 & 678.97 & 0.06 \\
\hline 25 & 116.75 & 38.04 & 35.37 & 4.76 & 2.53 & 23.84 & 10.92 & 29.94 & 12.32 & 710.11 & 0.13 \\
\hline 30 & 137.65 & 52.09 & 45.74 & 4.56 & 0.08 & 23.06 & 6.67 & 39.40 & 11.83 & 2111.24 & 0.22 \\
\hline 35 & 154.34 & 59.99 & 50.99 & 2.05 & 0.22 & 22.64 & 10.88 & 39.09 & 19.35 & 803.95 & 0.38 \\
\hline 40 & 176.75 & 63.99 & 55.97 & 1.33 & 0.41 & 24.63 & 9.63 & 39.98 & 26.97 & 881.32 & 0.56 \\
\hline 45 & 186.37 & 68.45 & 62.73 & 2.66 & 1.62 & 30.42 & 7.75 & 43.53 & 27.55 & 648.16 & 0.50 \\
\hline 50 & 204.21 & 67.83 & 62.03 & 3.79 & 2.72 & 19.18 & 7.89 & 47.35 & 33.52 & 1243.34 & 0.43 \\
\hline 55 & 213.58 & 70.60 & 49.49 & 6.40 & 4.03 & 19.83 & 9.57 & 49.46 & 31.74 & - & - \\
\hline 60 & 233.03 & 70.17 & 47.02 & 5.05 & 3.26 & 17.15 & 7.50 & 56.88 & 43.92 & - & - \\
\hline 65 & 242.39 & 70.56 & 52.88 & 5.16 & 2.44 & 18.46 & 8.94 & 61.03 & 48.41 & - & - \\
\hline 70 & 251.48 & 62.01 & 52.01 & 3.15 & 1.40 & 13.64 & 9.13 & 69.16 & 54.59 & - & - \\
\hline 75 & 260.00 & 63.21 & 55.52 & 3.98 & 1.98 & 14.69 & 9.71 & 69.11 & 50.36 & - & - \\
\hline 80 & 267.09 & 56.91 & 52.13 & 5.00 & 3.23 & 16.79 & 9.64 & 72.32 & 59.97 & - & - \\
\hline 85 & 277.22 & 50.72 & 35.71 & 4.95 & 4.07 & 12.71 & 8.03 & 76.61 & 46.19 & - & - \\
\hline 90 & 279.82 & 50.38 & 46.79 & 4.70 & 3.28 & 13.23 & 7.71 & 79.60 & 62.40 & - & - \\
\hline 95 & 281.12 & 47.78 & 38.69 & 5.15 & 3.42 & 15.19 & 8.99 & 75.94 & 51.85 & - & - \\
\hline 100 & 285.47 & 47.03 & 34.40 & 4.75 & 2.90 & 14.32 & 8.43 & 80.40 & 64.39 & - & - \\
\hline Av. & 142.20 & 34.15 & 27.34 & 5.66 & 2.17 & 15.17 & 6.21 & 33.43 & 21.67 & 373.07 & 0.13 \\
\hline
\end{tabular}

Table 5. Old/recent heuristics and the 1060-customer problem.

\begin{tabular}{rrrrrrrrrr}
\hline & \multirow{2}{*}{ CPU } & \multicolumn{2}{c}{ MALT } & \multicolumn{2}{c}{ FNS } & \multicolumn{2}{c}{ VNS-1 } & \multicolumn{2}{c}{ PROJ } \\
$m$ & \multicolumn{1}{c}{ Time } & Av. & \multicolumn{1}{c}{ Best } & Av. & Best & Av. & Best & Av. & Best \\
\hline 5 & 121.74 & 0.00 & 0.00 & 0.22 & 0.00 & 0.21 & 0.00 & 0.13 & 0.00 \\
10 & 242.04 & 0.03 & 0.01 & 0.10 & 0.00 & 0.10 & 0.00 & 1.09 & 0.04 \\
15 & 320.98 & 0.09 & 0.04 & 0.27 & 0.01 & 0.41 & 0.00 & 2.40 & 0.14 \\
20 & 377.04 & 1.17 & 1.09 & 2.17 & 1.72 & 2.10 & 1.71 & 3.66 & 0.36 \\
25 & 406.02 & 3.65 & 2.98 & 4.94 & 2.92 & 5.10 & 2.81 & 4.74 & 0.07 \\
30 & 507.45 & 8.20 & 7.28 & 7.73 & 5.89 & 8.51 & 5.44 & 5.11 & 1.17 \\
35 & 607.56 & 10.88 & 8.77 & 9.92 & 6.34 & 11.03 & 8.77 & 5.57 & 0.80 \\
40 & 679.96 & 14.92 & 13.19 & 12.77 & 10.58 & 15.68 & 11.18 & 7.46 & 1.53 \\
45 & 718.97 & 19.70 & 18.37 & 13.20 & 8.94 & 18.82 & 10.86 & 8.85 & 2.44 \\
50 & 740.57 & 23.75 & 22.36 & 13.73 & 10.09 & 23.37 & 15.81 & 10.20 & 2.14 \\
55 & 802.29 & 28.09 & 25.67 & 11.88 & 8.08 & 26.28 & 21.65 & 10.81 & 3.69 \\
60 & 855.83 & 32.38 & 30.19 & 13.20 & 10.15 & 26.31 & 20.59 & 11.79 & 2.83 \\
65 & 905.99 & 35.02 & 32.83 & 11.18 & 9.23 & 28.29 & 20.74 & 12.18 & 4.87 \\
70 & 956.30 & 38.59 & 37.17 & 10.69 & 7.38 & 32.75 & 23.22 & 12.59 & 3.82 \\
75 & 1005.61 & 39.75 & 37.29 & 10.02 & 7.60 & 31.77 & 23.33 & 13.21 & 4.22 \\
80 & 1020.64 & 41.77 & 38.00 & 9.46 & 3.77 & 31.13 & 27.05 & 13.05 & 4.49 \\
85 & 1028.75 & 42.79 & 40.71 & 7.87 & 4.48 & 31.19 & 24.24 & 13.42 & 5.08 \\
90 & 1044.53 & 42.75 & 39.10 & 7.16 & 3.83 & 32.67 & 23.60 & 13.44 & 5.51 \\
95 & 1084.29 & 43.59 & 35.57 & 4.04 & 2.08 & 34.31 & 28.30 & 13.60 & 5.41 \\
100 & 1092.74 & 43.59 & 37.26 & 3.37 & 2.02 & 33.33 & 23.34 & 13.20 & 5.02 \\
\hline Av. & 725.96 & 23.57 & 21.43 & 7.73 & 5.29 & 19.70 & 14.67 & 8.83 & 2.72 \\
\hline
\end{tabular}


Table 6. Descent DA heuristics in the 287-customer problem.

\begin{tabular}{|c|c|c|c|c|c|c|c|c|c|c|c|c|}
\hline \multirow[b]{2}{*}{$m$} & \multicolumn{3}{|c|}{ (DLU, AMU) } & \multicolumn{3}{|c|}{ (DLU, ASC) } & \multicolumn{3}{|c|}{ (DMP, ASC) } & \multicolumn{3}{|c|}{ (DSC, ASC) } \\
\hline & Av. & Best & Time & Av. & Best & Time & Av. & Best & Time & Av. & Best & Time \\
\hline 2 & 0.00 & 0.00 & 133.5 & 0.38 & 0.38 & 0.6 & 0.38 & 0.38 & 0.4 & 0.38 & 0.38 & 0.4 \\
\hline 3 & 0.00 & 0.00 & 99.7 & 0.14 & 0.00 & 1.1 & 0.17 & 0.00 & 0.6 & 0.17 & 0.00 & 0.6 \\
\hline 4 & 0.01 & 0.00 & 169.3 & 0.01 & 0.01 & 2.4 & 0.01 & 0.01 & 0.8 & 0.41 & 0.00 & 0.8 \\
\hline 5 & 0.01 & 0.01 & 227.0 & 0.01 & 0.01 & 4.0 & 0.01 & 0.01 & 1.1 & 1.11 & 0.01 & 1.0 \\
\hline 6 & 0.02 & 0.02 & 294.4 & 0.31 & 0.03 & 4.4 & 0.89 & 0.02 & 1.4 & 4.49 & 0.03 & 1.0 \\
\hline 7 & 0.03 & 0.02 & 296.1 & 0.03 & 0.03 & 6.4 & 0.83 & 0.02 & 1.5 & 1.49 & 0.03 & 1.2 \\
\hline 8 & 0.00 & 0.00 & 328.1 & 0.77 & 0.01 & 6.2 & 2.86 & 0.01 & 1.3 & 3.03 & 0.01 & 1.3 \\
\hline 9 & 0.29 & 0.00 & 297.8 & 0.51 & 0.00 & 6.8 & 1.84 & 0.50 & 1.3 & 2.90 & 0.08 & 1.2 \\
\hline 10 & 0.00 & 0.00 & 366.9 & 0.28 & 0.00 & 8.2 & 0.66 & 0.40 & 1.4 & 2.33 & 0.42 & 1.4 \\
\hline 11 & 0.23 & 0.00 & 337.6 & 0.76 & 0.15 & 10.5 & 1.78 & 0.47 & 1.4 & 3.42 & 0.49 & 1.3 \\
\hline 12 & 0.19 & 0.00 & 348.0 & 0.97 & 0.00 & 12.5 & 1.39 & 0.16 & 1.6 & 2.86 & 0.00 & 1.4 \\
\hline 13 & 0.35 & 0.00 & 378.0 & 0.83 & 0.11 & 12.5 & 1.66 & 0.32 & 1.5 & 3.45 & 0.32 & 1.3 \\
\hline 14 & 0.42 & 0.01 & 367.6 & 1.23 & 0.27 & 16.0 & 1.43 & 0.20 & 1.7 & 3.84 & 1.03 & 1.3 \\
\hline 15 & 0.53 & 0.05 & 369.7 & 2.18 & 0.65 & 15.3 & 2.67 & 0.76 & 1.6 & 3.16 & 1.56 & 1.5 \\
\hline 16 & 0.61 & 0.18 & 382.9 & 1.21 & 0.34 & 14.7 & 2.39 & 0.66 & 1.5 & 2.89 & 0.95 & 1.4 \\
\hline 17 & 0.43 & 0.09 & 470.8 & 1.03 & 0.23 & 19.6 & 3.40 & 0.76 & 1.5 & 4.63 & 2.72 & 1.4 \\
\hline 18 & 0.09 & 0.01 & 535.7 & 0.96 & 0.06 & 23.6 & 2.89 & 0.76 & 1.9 & 4.81 & 2.43 & 1.5 \\
\hline 19 & 0.12 & 0.01 & 621.2 & 1.65 & 0.34 & 23.1 & 2.67 & 0.34 & 2.0 & 5.18 & 2.52 & 1.7 \\
\hline 20 & 0.11 & 0.01 & 538.3 & 2.86 & 0.03 & 21.6 & 3.03 & 0.93 & 1.9 & 3.29 & 1.45 & 1.9 \\
\hline 25 & 0.31 & 0.00 & 675.3 & 0.68 & 0.01 & 37.1 & 2.32 & 0.61 & 3.0 & 6.68 & 1.46 & 2.2 \\
\hline 30 & 0.08 & 0.03 & 693.9 & 0.36 & 0.03 & 50.0 & 0.86 & 0.05 & 3.4 & 5.37 & 2.74 & 2.8 \\
\hline 35 & 0.02 & 0.01 & 809.3 & 0.03 & 0.01 & 66.6 & 1.41 & 0.01 & 3.8 & 4.40 & 1.35 & 3.5 \\
\hline 40 & 0.10 & 0.01 & 1081.1 & 3.44 & 0.01 & 93.1 & 3.94 & 1.16 & 4.0 & 5.50 & 1.50 & 4.2 \\
\hline 45 & 0.15 & 0.01 & 1175.0 & 3.96 & 0.01 & 113.5 & 4.00 & 1.53 & 3.8 & 6.15 & 2.93 & 4.4 \\
\hline 50 & 0.02 & 0.00 & 1462.4 & 3.23 & 0.07 & 142.3 & 2.97 & 2.11 & 4.9 & 5.99 & 1.23 & 4.9 \\
\hline 55 & 0.03 & 0.00 & 1721.0 & 3.87 & 0.00 & 213.3 & 3.93 & 1.58 & 6.2 & 8.78 & 5.31 & 5.7 \\
\hline 60 & 0.15 & 0.01 & 1905.9 & 0.23 & 0.01 & 275.5 & 2.43 & 1.19 & 7.1 & 8.47 & 1.85 & 6.2 \\
\hline 65 & 0.09 & 0.01 & 1934.9 & 0.61 & 0.01 & 314.6 & 3.48 & 0.83 & 7.2 & 13.94 & 8.08 & 5.5 \\
\hline 70 & 0.07 & 0.00 & 2003.5 & 0.60 & 0.06 & 363.9 & 2.70 & 0.76 & 7.9 & 9.01 & 3.95 & 6.7 \\
\hline 75 & 0.08 & 0.04 & 2462.3 & 1.18 & 0.08 & 452.1 & 1.62 & 0.12 & 10.3 & 10.57 & 6.77 & 7.6 \\
\hline 80 & 0.18 & 0.05 & 2444.6 & 0.51 & 0.12 & 519.1 & 3.14 & 1.59 & 9.4 & 8.22 & 4.40 & 8.3 \\
\hline 85 & 0.14 & 0.04 & 2865.1 & 0.66 & 0.04 & 601.4 & 2.82 & 1.09 & 10.0 & 7.35 & 3.70 & 9.4 \\
\hline 90 & 0.06 & 0.01 & 3190.0 & 3.47 & 0.01 & 656.9 & 3.07 & 0.11 & 11.6 & 9.60 & 5.14 & 10.1 \\
\hline 95 & 0.11 & 0.02 & 3171.0 & 0.15 & 0.02 & 846.5 & 2.28 & 0.55 & 13.4 & 10.05 & 5.49 & 10.8 \\
\hline 100 & 0.01 & 0.00 & 3605.0 & 1.55 & 0.00 & 925.8 & 3.58 & 1.54 & 12.2 & 8.54 & 3.95 & 11.5 \\
\hline Av. & 0.14 & 0.02 & 1078.9 & 1.16 & 0.09 & 168.0 & 2.16 & 0.62 & 4.1 & 5.21 & 2.12 & 3.6 \\
\hline
\end{tabular}

the best result. Computation times are totals for the $10 \mathrm{de}-$ scents. Referring to the best solutions, we first observe that the drop least useful, add most useful strategy (DLU, AMU) worked very well. The percent deviation from optimal is low irrespective of $m$, and regularly, the optimal solution itself is obtained. Note, however, that the computational times are much higher than for the other DA heuristics. This is attributed to the excessive number of Cooper iterations carried out between adjacent moves in the solution space.

A compromise between quality and CPU time is obtained with (DLU, ASC) because the second-closest criterion is fast to implement. (Also AMU takes much longer than DLU, especially when $m$ is small.) The fastest procedures are given by (DMP, ASC) and (DSC, ASC) because optimal relocation of facilities is not carried out during the drop and add phases. The listed computation times for (DMP, ASC) and (DSC, ASC) are substantially less than those for MALT. Yet, a comparison shows for example that (DMP, ASC) performs significantly better than MALT and the "parameterless" VNS-1.
Table 7 presents a summary of results for four local search heuristics using relocation neighbourhood structures. The values listed here are combined average percentage deviations and CPU times over the same sets of values of $m$ reported in the previous tables. The columns have the same interpretation as in Table 6.

The first two heuristics in Table 7 are the "fast" versions of drop/add reported in Table 6. The next heuristic (referred to as $\mathrm{CH}$ for interchange) considers all possible location interchanges of a single facility from its current position to an unoccupied fixed point. One iteration of Cooper's algorithm (allocate-locate) is run only at the best neighbourhood point to save computation time. The fourth heuristic PM-2 is a discrete version, where the facilities remain at fixed points during the interchange process. When no further improvement can be made (the current solution is a local minimum in its neighbourhood), one iteration of Cooper's algorithm is performed as in $\mathrm{CH}$.

Comparing the four relocation heuristics in Table 7, we see that no one method dominates the others. DA-2 obtains 
Table 7. Summary results for local descent heuristics with Relocation neighbourhoods.

\begin{tabular}{|c|c|c|c|c|c|c|c|c|c|c|c|c|}
\hline \multirow[b]{2}{*}{$\mathrm{Pb}$. } & \multicolumn{3}{|c|}{$\mathrm{DA}-1 \equiv(\mathrm{DMP}, \mathrm{ASC})$} & \multicolumn{3}{|c|}{$\mathrm{DA}-2 \equiv(\mathrm{DSC}, \mathrm{ASC})$} & \multicolumn{3}{|c|}{$\mathrm{CH}$} & \multicolumn{3}{|c|}{ PM-2 } \\
\hline & Av. & Best & Time & Av. & Best & Time & Av. & Best & Time & Av. & Best & Time \\
\hline 50 & 7.06 & 2.07 & 0.07 & 0.98 & 0.21 & 0.15 & 1.18 & 0.11 & 0.17 & 0.58 & 0.02 & 0.04 \\
\hline 287 & 2.16 & 0.62 & 4.13 & 5.21 & 2.12 & 3.65 & 0.26 & 0.04 & 3.35 & 0.28 & 0.07 & 1.33 \\
\hline 654 & 6.64 & 4.02 & 18.66 & 0.58 & 0.36 & 32.42 & 0.95 & 0.53 & 29.93 & 0.29 & 0.01 & 8.11 \\
\hline 1060 & 2.83 & 1.57 & 85.40 & 0.48 & 0.21 & 115.09 & 1.05 & 0.67 & 93.29 & 0.67 & 0.21 & 71.03 \\
\hline Av. & 4.67 & 2.07 & 27.07 & 1.81 & 0.73 & 37.83 & 0.86 & 0.34 & 31.69 & 0.45 & 0.08 & 20.13 \\
\hline
\end{tabular}

better results than DA-1 in three of the four problem sets, but not in the 287-customer set. Interestingly, the same pattern occurs between PM-2 and $\mathrm{CH}$. As a group, $\mathrm{CH}$ and PM-2 obtain better solutions than the DA heuristics in all problem sets. However, DA-2 obtains the best average deviation in the 1060-customer set and outperforms $\mathrm{CH}$ here and in the 654-customer set. More importantly, we observe that these new local search heuristics are very efficient compared with the earlier methods reported in Tables 2 to 5 . With the exception of VNS-1 in Table 2 (50-customer set), large net improvements are obtained over the earlier methods in a small fraction of the CPU time.

\section{New Heuristics}

Tables 8 to 11 report on our new heuristics. These consist of a genetic algorithm (GA) and the latest versions of Tabu search and variable neighbourhood search, using a relocation neighbourhood structure in place of the previous reallocation structure. The parameter settings used in GA are $N=15$ for the initial population, and $Q_{\max }=30$ for population size. The first Tabu search procedure (TS-2) uses a drop/add neighbourhood and DA-2 strategy, while the second (TS-3) uses the interchange neighbourhood of CH. In both cases, a Tabu list is maintained of the last 15 fixedpoint insertions. The same neighbourhood structures are utilized again in the variable neighbourhood searches VNS-2 and VNS-3, while VNS-4 borrows the discrete interchange structure of PM-2. In the three VNS versions, the maximum neighbourhood size, $k_{\max }$, is set equal to $m$, and one point is randomly chosen in each neighbourhood. Only one iteration of Cooper's algorithm is performed at a selected neighbourhood point to make efficient use of CPU time. Note that these CPU times are not recorded in the tables because they were set to the execution times previously obtained for MALT.

Table 8. New heuristics and the 50-customer problem.

\begin{tabular}{|c|c|c|c|c|c|c|c|c|c|c|c|c|}
\hline \multirow[b]{3}{*}{$m$} & & & \multicolumn{4}{|c|}{ DA-2 } & \multicolumn{4}{|c|}{$\mathrm{CH}$} & \multirow{2}{*}{\multicolumn{2}{|c|}{$\begin{array}{l}\text { P-MED } \\
\text { VNS-4 }\end{array}$}} \\
\hline & \multicolumn{2}{|c|}{ GA } & \multicolumn{2}{|c|}{ TS-2 } & \multicolumn{2}{|c|}{ VNS-2 } & \multicolumn{2}{|c|}{ TS-3 } & \multicolumn{2}{|c|}{ VNS-3 } & & \\
\hline & Av. & Best & Av. & Best & Av. & Best & Av. & Best & Av. & Best & Av. & Best \\
\hline 2 & 0.00 & 0.00 & 0.92 & 0.00 & 0.08 & 0.00 & 0.01 & 0.00 & 0.00 & 0.00 & 0.01 & 0.00 \\
\hline 3 & 0.00 & 0.00 & 0.93 & 0.64 & 0.24 & 0.00 & 0.02 & 0.00 & 0.00 & 0.00 & 0.00 & 0.00 \\
\hline 4 & 0.00 & 0.00 & 0.12 & 0.01 & 0.00 & 0.00 & 0.00 & 0.00 & 0.00 & 0.00 & 0.00 & 0.00 \\
\hline 5 & 0.01 & 0.00 & 0.19 & 0.01 & 0.11 & 0.00 & 0.33 & 0.00 & 0.00 & 0.00 & 0.19 & 0.00 \\
\hline 6 & 0.04 & 0.00 & 0.61 & 0.21 & 0.00 & 0.00 & 0.00 & 0.00 & 0.00 & 0.00 & 0.00 & 0.00 \\
\hline 7 & 0.07 & 0.00 & 0.02 & 0.00 & 0.00 & 0.00 & 1.34 & 0.00 & 0.00 & 0.00 & 0.02 & 0.00 \\
\hline 8 & 0.23 & 0.00 & 0.19 & 0.00 & 0.00 & 0.00 & 0.95 & 0.00 & 0.00 & 0.00 & 0.00 & 0.00 \\
\hline 9 & 0.68 & 0.00 & 0.57 & 0.00 & 0.00 & 0.00 & 0.78 & 0.00 & 0.00 & 0.00 & 0.00 & 0.00 \\
\hline 10 & 0.42 & 0.00 & 0.01 & 0.00 & 0.00 & 0.00 & 1.43 & 0.00 & 0.00 & 0.00 & 0.00 & 0.00 \\
\hline 11 & 1.24 & 0.00 & 0.47 & 0.00 & 0.00 & 0.00 & 1.69 & 0.00 & 0.00 & 0.00 & 0.00 & 0.00 \\
\hline 12 & 1.72 & 0.00 & 0.40 & 0.00 & 0.00 & 0.00 & 2.06 & 0.00 & 0.00 & 0.00 & 0.00 & 0.00 \\
\hline 13 & 1.47 & 0.00 & 0.68 & 0.00 & 0.00 & 0.00 & 0.34 & 0.00 & 0.00 & 0.00 & 0.03 & 0.00 \\
\hline 14 & 2.79 & 0.41 & 0.46 & 0.00 & 0.00 & 0.00 & 1.76 & 0.00 & 0.00 & 0.00 & 0.00 & 0.00 \\
\hline 15 & 3.10 & 0.16 & 0.67 & 0.00 & 0.00 & 0.00 & 0.36 & 0.00 & 0.00 & 0.00 & 0.00 & 0.00 \\
\hline 16 & 1.71 & 0.11 & 0.86 & 0.26 & 0.03 & 0.00 & 1.37 & 0.52 & 0.00 & 0.00 & 0.00 & 0.00 \\
\hline 17 & 3.99 & 0.44 & 1.31 & 0.00 & 0.04 & 0.00 & 0.66 & 0.00 & 0.00 & 0.00 & 0.00 & 0.00 \\
\hline 18 & 4.56 & 0.48 & 1.45 & 0.00 & 0.03 & 0.00 & 0.71 & 0.00 & 0.00 & 0.00 & 0.03 & 0.00 \\
\hline 19 & 6.25 & 2.27 & 1.27 & 0.09 & 0.01 & 0.00 & 0.46 & 0.00 & 0.00 & 0.00 & 0.00 & 0.00 \\
\hline 20 & 4.41 & 0.52 & 1.37 & 0.68 & 0.00 & 0.00 & 0.84 & 0.00 & 0.00 & 0.00 & 0.07 & 0.00 \\
\hline 21 & 4.91 & 1.56 & 0.91 & 0.00 & 0.00 & 0.00 & 0.84 & 0.13 & 0.00 & 0.00 & 0.08 & 0.00 \\
\hline 22 & 4.42 & 0.97 & 1.14 & 0.54 & 0.05 & 0.00 & 0.70 & 0.00 & 0.00 & 0.00 & 0.09 & 0.00 \\
\hline 23 & 6.66 & 1.82 & 1.23 & 0.00 & 0.12 & 0.00 & 0.91 & 0.24 & 0.02 & 0.00 & 0.09 & 0.00 \\
\hline 24 & 8.39 & 3.79 & 1.33 & 0.37 & 0.02 & 0.00 & 0.98 & 0.00 & 0.00 & 0.00 & 0.10 & 0.00 \\
\hline 25 & 9.73 & 6.07 & 2.07 & 0.00 & 0.00 & 0.00 & 0.42 & 0.00 & 0.00 & 0.00 & 0.08 & 0.00 \\
\hline iv. & 2.78 & 0.78 & 0.80 & 0.12 & 0.03 & 0.00 & 0.79 & 0.04 & 0.00 & 0.00 & 0.03 & 0.00 \\
\hline
\end{tabular}


Table 9. New heuristics and the 287-customer problem.

\begin{tabular}{|c|c|c|c|c|c|c|c|c|c|c|c|c|}
\hline \multirow[b]{3}{*}{$m$} & & & \multicolumn{4}{|c|}{ DA-2 } & \multicolumn{4}{|c|}{$\mathrm{CH}$} & \multirow{2}{*}{\multicolumn{2}{|c|}{$\begin{array}{l}\text { P-MED } \\
\text { VNS-4 }\end{array}$}} \\
\hline & \multicolumn{2}{|c|}{ GA } & \multicolumn{2}{|c|}{ TS-2 } & \multicolumn{2}{|c|}{ VNS-2 } & \multicolumn{2}{|c|}{ TS-3 } & \multicolumn{2}{|c|}{ VNS-3 } & & \\
\hline & Av. & Best & Av. & Best & Av. & Best & Av. & Best & Av. & Best & Av. & Best \\
\hline 2 & 0.04 & 0.00 & 0.07 & 0.00 & 0.58 & 0.24 & 0.02 & 0.00 & 0.00 & 0.00 & 0.00 & 0.00 \\
\hline 3 & 0.00 & 0.00 & 0.05 & 0.00 & 0.03 & 0.00 & 0.00 & 0.00 & 0.00 & & 0.00 & 0.00 \\
\hline 4 & 0.00 & 0.00 & 0.02 & 0.01 & 0.00 & 0.00 & 0.00 & 0.00 & 0.00 & & 0.00 & 0.00 \\
\hline 5 & 0.04 & 0.00 & 0.09 & 0.01 & 0.01 & 0.01 & 0.01 & 0.01 & 0.01 & 0.01 & 0.01 & 0.01 \\
\hline 6 & 0.53 & 0.00 & 2.56 & 0.02 & 0.02 & 0.02 & 0.02 & 0.02 & 0.02 & 0.02 & 0.02 & 0.02 \\
\hline 7 & 0.21 & 0.00 & 3.46 & 0.03 & 0.04 & 0.03 & 0.03 & 0.03 & 0.02 & 0.02 & 0.03 & 0.03 \\
\hline 8 & 0.35 & 0.00 & 3.28 & 0.09 & 0.01 & 0.00 & 0.01 & 0.01 & 0.01 & 0.00 & 0.28 & 0.01 \\
\hline 9 & 0.38 & 0.00 & 4.23 & 0.01 & 0.04 & 0.00 & 0.01 & 0.00 & 0.00 & 0.00 & 0.33 & 0.01 \\
\hline 10 & 0.57 & 0.00 & 3.71 & 0.00 & 0.17 & 0.01 & 0.23 & 0.00 & 0.00 & 0.00 & 0.23 & 0.00 \\
\hline 11 & 0.58 & 0.00 & 1.21 & 0.00 & 0.16 & 0.00 & 0.03 & 0.00 & 0.00 & 0.00 & 0.23 & 0.00 \\
\hline 12 & 0.82 & 0.18 & 2.41 & 0.14 & 0.39 & 0.14 & 0.09 & 0.00 & 0.06 & 0.00 & 0.24 & 0.00 \\
\hline 13 & 0.61 & 0.00 & 3.06 & 0.31 & 0.53 & 0.34 & 0.10 & 0.00 & 0.03 & 0.00 & 0.36 & 0.00 \\
\hline 14 & 0.81 & 0.39 & 1.53 & 0.22 & 0.43 & 0.22 & 0.21 & 0.01 & 0.01 & 0.01 & 0.31 & 0.20 \\
\hline 15 & 0.93 & 0.17 & 2.58 & 0.51 & 0.32 & 0.01 & 0.11 & 0.01 & 0.01 & 0.01 & 0.13 & 0.10 \\
\hline 16 & 1.41 & 0.0 & 2 & 0.14 & 0.64 & 0.11 & 0.26 & 0.01 & 0.01 & 0 & 0.14 & 0.10 \\
\hline 17 & 1.23 & 0.79 & 3. & 0.85 & 0.96 & 0.63 & 0.56 & 0.01 & 0.09 & 0.01 & 0.17 & 0.01 \\
\hline 18 & 1.24 & 0.42 & 2.78 & 0.38 & 0.76 & 0.17 & 0.29 & 0.06 & 0.06 & 0.01 & 0.08 & 0.01 \\
\hline 19 & 1.96 & 0.84 & 3.88 & 2.23 & 1.03 & 0.16 & 0.53 & 0.07 & 0.06 & 0.01 & 0.09 & 0.01 \\
\hline 20 & 1.59 & 0.07 & 4.09 & 0.42 & 1.16 & 0.11 & 0.41 & 0.13 & 0.05 & 0.01 & 0.06 & 0.01 \\
\hline 25 & 2.33 & 0.01 & 3.75 & 1.50 & 1.13 & 0.16 & 0.36 & 0.01 & 0.01 & 0.00 & 0.01 & 0.00 \\
\hline 30 & 3.91 & 0.53 & 6.66 & 1.44 & 1.92 & 0.31 & 0.57 & 0.03 & 0.03 & 0.03 & 0.03 & 0.03 \\
\hline 35 & 4.09 & 0.74 & 5.77 & 1.56 & 2.59 & 0.61 & 0.12 & 0.01 & 0.01 & 0.01 & 0.03 & 0.01 \\
\hline 40 & 5.61 & 4.19 & 5.45 & 1.90 & 3.11 & 0.92 & 0.54 & 0.01 & 0.19 & 0.01 & 0.09 & 0.01 \\
\hline 45 & 5.25 & 2.60 & 4.81 & 0.67 & 3.34 & 0.68 & 0.18 & 0.01 & 0.01 & 0.01 & 0.04 & 0.01 \\
\hline 50 & 7.11 & 4.53 & 4.78 & 1.35 & 2.95 & 1.31 & 0.17 & 0.00 & 0.00 & 0.00 & 0.00 & 0.00 \\
\hline 55 & 8.69 & 5.76 & & 3.70 & 4.74 & 1.65 & 0.20 & 0.00 & 0.12 & 0 & 0.02 & 0.00 \\
\hline 60 & 8.90 & 5.8 & & 0.29 & 4.53 & & & & & & 2 & 0.01 \\
\hline 65 & 10.04 & 4.8 & 6.53 & 2.72 & 4.75 & 1.65 & 0.29 & 0.01 & 0.03 & 0.01 & 0.12 & 0.01 \\
\hline 70 & 11.91 & 6.65 & 9.01 & 5.62 & 6.86 & 4.05 & 0.75 & 0.03 & 0.07 & 0.03 & 0.08 & 0.03 \\
\hline 75 & 11.57 & 7.68 & 7.38 & 3.00 & 4.59 & 2.45 & 0.25 & 0.04 & 0.09 & 0.04 & 0.16 & 0.05 \\
\hline 80 & 12.00 & 4.96 & 5.58 & 2.75 & 3.59 & 2.34 & 0.29 & 0.04 & 0.08 & 0.04 & 0.22 & 0.06 \\
\hline 85 & 12.39 & 8.35 & 6.50 & 3.94 & 4.66 & 2.49 & 0.34 & 0.04 & 0.04 & 0.04 & 0.13 & 0.05 \\
\hline 90 & 15.38 & 10.51 & 8.87 & 4.65 & 5.53 & 3.09 & 0.19 & 0.02 & 0.02 & 0.01 & 0.09 & 0.02 \\
\hline 95 & 14.38 & 10.44 & 6.94 & 2.57 & 4.17 & 2.43 & 0.06 & 0.02 & 0.02 & 0.02 & 0.18 & 0.02 \\
\hline 100 & 14.29 & 10.30 & 6.03 & 3.42 & 3.89 & 1.52 & 0.06 & 0.01 & 0.02 & 0.01 & 0.10 & 0.01 \\
\hline Av. & 4.60 & 2.59 & 4.13 & 1.33 & 1.99 & 0.81 & 0.22 & 0.02 & 0.04 & 0.01 & 0.12 & 0.02 \\
\hline
\end{tabular}

Referring to Tables 8 to 11, the following observations are made.

OBSERVATIOn 1. GA performs very well over the lower range of $m$ values in all four problem sets. However, the percentage deviation tends to increase with $m$. This may be attributed to an exponentially increasing number of local minima and the fact that GA has time to visit only a small number of them.

Observation 2. TS-3 outperforms TS-2 in Tables 8 and 9 , but the reverse is true in Tables 10 and 11. Based on these limited data, we might infer that the interchange neighbourhood $(\mathrm{CH})$ is better suited for smaller problems, while the drop/add (DA-2) should be used for larger instances. This may be because of the longer CPU time required to make a move in the interchange neighbourhood, which results in fewer iterations. Also note that the best TS heuristic outperforms $\mathrm{GA}$ in each table.
ObSERVATION 3. A similar relation is observed between VNS-2 and VNS-3. Once again, the drop/add neighbourhood appears to be a better choice for larger problems. We might infer that the DA strategy in VNS-2, although more time consuming, has more success finding descent directions in large problems as compared with the random selection of neighbourhood points in VNS-3. Hence, VNS-2 would be able to make more descent moves in the allotted $\mathrm{CPU}$ time.

ObServation 4. Comparing TS-2 with VNS-2 and TS-3 with VNS-3, we observe that better results are obtained by the variable neighbourhood approach. However, TS-2 is competitive in Tables 10 and 11, where it outperforms VNS-2 and VNS-3 in a few cases.

ObSERVATIOn 5. VNS-3 is clearly the best overall method in Tables 8 and 9, but VNS-4 takes over in Tables 10 and 11. The advantage with VNS-4 pertains to its $p$-Median 
Table 10. New heuristics and the 654-customer problem.

\begin{tabular}{|c|c|c|c|c|c|c|c|c|c|c|c|c|}
\hline \multirow[b]{3}{*}{$m$} & & & \multicolumn{4}{|c|}{ DA-2 } & \multicolumn{4}{|c|}{$\mathrm{CH}$} & \multirow{2}{*}{\multicolumn{2}{|c|}{$\begin{array}{l}\text { P-MED } \\
\text { VNS-4 }\end{array}$}} \\
\hline & \multicolumn{2}{|c|}{ GA } & \multicolumn{2}{|c|}{ TS-2 } & \multicolumn{2}{|c|}{ VNS-2 } & \multicolumn{2}{|c|}{ TS-3 } & \multicolumn{2}{|c|}{ VNS-3 } & & \\
\hline & Av. & Best & Av. & Best & Av. & Best & Av. & Best & Av. & Best & Av. & Best \\
\hline 2 & 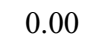 & 0.00 & 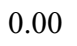 & 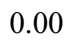 & & & 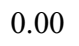 & 0.00 & 00 & & .00 & 0.00 \\
\hline 3 & & & 17 & 0.00 & 13 & & & 0.00 & .00 & & .00 & 0.00 \\
\hline 4 & 0.00 & 0.00 & 0.03 & 0.02 & 0.07 & & 0.00 & 0.00 & 0.00 & & & 0.00 \\
\hline 5 & 0.00 & & & & & & 0.00 & & 0.00 & & & \\
\hline 6 & 0.57 & 0.0 & & 0 . & 0.00 & & 0.00 & 0.00 & 0.00 & & 0.00 & 000 \\
\hline 7 & 0.0 & & & & & & & & & & & م \\
\hline 8 & & & & & & & & & & & & 00 \\
\hline 9 & & & & & & & & & & & & 00 \\
\hline 10 & & & & & & & & & & & & 00 \\
\hline 11 & 2.1 & & & & & & & & & & 00 & 0.00 \\
\hline 12 & 2.10 & 000 & 000 & 0.00 & & & 0.00 & 0 & 0.00 & & 0.15 & 0.00 \\
\hline 13 & 1.07 & & & 0.01 & 009 & & 0.60 & 0.19 & 0.01 & & 0.11 & 0.00 \\
\hline 14 & 0.52 & 0 . & 0.04 & 0 . & 12 & & 0.55 & 0.52 & 0.01 & & 0.01 & 0.00 \\
\hline 15 & 1.55 & 0.0 & 0 & 0.04 & 0.05 & 0.01 & 0.54 & 0.53 & 0.02 & 0.01 & 0.02 & 0.00 \\
\hline 20 & 0.86 & 0.0 & & 0.02 & & & 0.30 & 0.28 & 0.06 & & & 0.00 \\
\hline 25 & 3.01 & & 5 & 0.02 & & & 0.55 & 0 . & 0.21 & & 7 & 0.01 \\
\hline 30 & 5.4 & 0.0 & & 0.01 & & & & & 0.06 & & & 0.01 \\
\hline 35 & 6.5 & & & & & & & & 2 & & & 0.01 \\
\hline 40 & 7.6 & & & & & & & &  & & & 0.04 \\
\hline 45 & 6 & & 9 & 0 & 6 & & & 1 & 3 & 3 & 0.50 & 0.13 \\
\hline 50 & 30 & & & & & & 2.88 & 2.22 & 0 & & 0.55 & 0.11 \\
\hline 55 & 12.25 & & & & & & 2.48 & 0.96 & 0.92 & & 0.29 & 0.00 \\
\hline 60 & 14.49 & & & & & & 1.78 & 0.92 & 1.05 & & 0.28 & 0.05 \\
\hline 65 & 18.32 & 12.57 & & & & & 1.59 & 0.39 & 0.99 & & 0.40 & 0.04 \\
\hline 70 & 20.22 & 14.34 & 0.48 & 0.17 & 0.31 & & 0.60 & 0.25 & 0.33 & 0.11 & 0.39 & 0.20 \\
\hline 75 & & & & & & & & & 0.35 & & 0.51 & 0.31 \\
\hline 80 & 24.52 & & 0.87 & & & & 1.74 & 1.29 & 1.10 & & 1.26 & 0.71 \\
\hline 85 & 24.54 & 18.03 & 1.10 & 0.73 & 0.9 & 0. & 1.38 & 1.04 & 0.96 & 0.64 & 1.06 & 0.73 \\
\hline 90 & 27.73 & & & 0.64 & 0.8 & & 1.23 & 0.27 & 0.71 & 0.25 & 0.58 & 0.37 \\
\hline 95 & 26.99 & 20.4 & 0.90 & 0.5 & 0 & & 1.40 & 0.96 & 0.74 & 0.51 & 0.55 & 0.28 \\
\hline 100 & 30.79 & 25.82 & 0.47 & 0.11 & 0.42 & 0.11 & 1.32 & 0.64 & 0.46 & 0.15 & 0.49 & 0.14 \\
\hline Av. & .73 & 5.79 & 0.30 & 0.17 & 0.24 & 0.14 & 0.82 & 0.46 & 0.34 & 0.17 & 0.25 & 0.10 \\
\hline
\end{tabular}

neighbourhood structure. Because the facilities are kept at fixed-point locations, VNS-4 is able to evaluate neighbourhood points extremely quickly. Only when a descent move is made does the algorithm locate the facilities in continuous space to obtain a candidate solution. The greater number of visits through the neighbourhoods with VNS-4 appears to be a critical factor in large problems.

\section{CONCLUSIONS}

An extensive empirical study is presented of heuristic methods for solving the multisource Weber problem. Included are several new methods that have not been reported previously. An important aspect of the current work is that it considers much larger problem sizes than previously investigated in the literature. Thus, we are able to show that the state of the art heuristics tend to deteriorate in performance with increasing problem size, sometimes in a disastrous fashion.
The new heuristics presented here obtained excellent results, which are far superior to the solutions found by the existing methods. Deviations from the best known solutions of less than $0.1 \%$ are consistently reported by the new methods over all problem sets. The fact is made more remarkable in view of the restricted CPU time. Thus, we may claim that the state of the art is advanced.

Some general conclusions are inferred from the results of this study.

1. Relocation-based methods (drop/add or interchange) are more efficient than their counterpart reallocation-based methods. That is, better solutions are obtained in general in the same CPU time, when local or variable neighbourhood searches are conducted with a relocation neighbourhood structure. One reason may be the fact that the neighbourhood points in the relocation structure correspond to Voronoi partitions of the customer set, but not so for reallocations.

2. The variable neighbourhood concept can be effectively used to obtain superior solutions. We may view the variable 
Table 11. New heuristics and the 1060-customer problem.

\begin{tabular}{|c|c|c|c|c|c|c|c|c|c|c|c|c|}
\hline \multirow[b]{3}{*}{$m$} & & & \multicolumn{4}{|c|}{ DA-2 } & \multicolumn{4}{|c|}{$\mathrm{CH}$} & \multirow{2}{*}{\multicolumn{2}{|c|}{$\begin{array}{l}\text { P-MED } \\
\text { VNS-4 }\end{array}$}} \\
\hline & \multicolumn{2}{|c|}{ GA } & \multicolumn{2}{|c|}{ TS-2 } & \multicolumn{2}{|c|}{ VNS-2 } & \multicolumn{2}{|c|}{ TS-3 } & \multicolumn{2}{|c|}{ VNS-3 } & & \\
\hline & Av. & Best & Av. & Best & Av. & Best & Av. & Best & Av. & Best & Av. & Best \\
\hline 5 & .00 & & & 08 & 2 & & 0.00 & 0.00 & 0.02 & & 0.00 & 08 \\
\hline 10 & .03 & & & 5 & & & 04 & 00 & 1 & & .01 & 00 \\
\hline 15 & 0.06 & 0.0 & & 0.18 & & & 20 & 01 & & & .04 & 01 \\
\hline 20 & 0.25 & 0.0 & & 0.05 & & & 0.78 & 0.53 & & & .06 & 0.00 \\
\hline 25 & 0.26 & 0.09 & & 0.04 & 0.07 & & 0.45 & 0.02 & 0.09 & 0.01 & 0.17 & 0.02 \\
\hline 30 & 0.65 & 0.04 & 0.37 & 0.31 & 0.30 & 0.1 & 0.79 & 0.22 & 0.15 & 0.03 & 0.06 & 0.00 \\
\hline 35 & 1.04 & 0.35 & 0.19 & 0.02 & 0.15 & 0.02 & 1.89 & 1.15 & 0.46 & 0.11 & 0.09 & 0.00 \\
\hline 40 & 1.36 & 0.77 & 0.12 & 0.01 & 0.16 & 0.06 & 2.11 & 1.30 & 0.67 & 0.38 & 0.10 & 0.00 \\
\hline 45 & 1.56 & 0.69 & 0.13 & 0.02 & 0.17 & 0.00 & 1.52 & 0.94 & 0.45 & & 0.19 & 0.01 \\
\hline 50 & 2.48 & 1.52 & 0.22 & 0.05 & 0.16 & 0.0 & 1.77 & 1.29 & 0.66 & 0.45 & 0.21 & 0.08 \\
\hline 55 & 1.97 & 0.69 & 0.13 & 0.02 & 0.10 & 0.0 & 0.91 & 0.21 & 0.16 & 0. & 0.21 & 0.00 \\
\hline 60 & 2.42 & 0.8 & 0.15 & 0.02 & 0.10 & 0. & 0.92 & 0.51 & 0.21 & 0. & 0.07 & 0.00 \\
\hline 65 & 2.19 & 0.9 & & 0.09 & 0 & 0. & 0.96 & 0.51 & 0 . & 0 & 0.17 & 0.00 \\
\hline 70 & 3.49 & 2.0 & 0.24 & 0.05 & 0.15 & 0.06 & 0.95 & 0.57 & 0.29 & 0.11 & 0.27 & 0.02 \\
\hline 75 & 3.72 & 1.8 & 0 & 0.00 & 0.13 & 0.03 & 0.84 & 0.61 & 0.29 & 0.01 & 0.20 & 0.00 \\
\hline 80 & 4.35 & 3.21 & 0.37 & 0.06 & 0.13 & 0.03 & 0.71 & 0.21 & 0.22 & 0.01 & 0.28 & 0.00 \\
\hline 85 & 4.18 & 1.86 & 0.30 & 0.13 & 0.13 & 0.00 & 0.65 & 0.25 & 0.19 & 0.10 & 0.24 & 0.07 \\
\hline 90 & 4.24 & 3.14 & 0.30 & 0.15 & 0.12 & 0.04 & 0.87 & 0.58 & 0.41 & 0.16 & 0.31 & 0.01 \\
\hline 95 & 4.49 & 3.49 & 0.23 & 0.06 & 0.13 & 0.00 & 0.77 & 0.55 & 0.33 & 0.13 & 0.23 & 0.01 \\
\hline 100 & 4.71 & 3.83 & 0.42 & 0.11 & 0.30 & 0.10 & 0.92 & 0.47 & 0.47 & 0.28 & 0.33 & 0.07 \\
\hline Av. & 2.17 & 1.27 & 0.28 & 0.13 & 0.21 & 0.08 & 0.90 & 0.49 & 0.26 & 0.10 & 0.16 & 0.02 \\
\hline
\end{tabular}

neighbourhood search (VNS) as a "shaking" process, where movement to a successive neighbourhood corresponds to a harder shake. Unlike random restart, which moves from the current solution to any point (uncontrolled shaking) typically far away, VNS allows a controlled increase in the level of the shake.

3. Comparison of the new heuristics suggests that no one method is best in all cases. Issues to consider in the design of an algorithm include the type of neighbourhood (e.g., drop/add or interchange, discrete or continuous facility locations), the amount of shaking to permit, when to conduct local searches at neighbourhood points and by what method, and whether or not to permit ascent moves. The variation of strategies is limitless in terms of shaking and local search, and parameter settings. Future studies may establish general guidelines for choosing the 'best' algorithm as a function of problem size ( $m$ and $n$ ).

\section{ACKNOWLEDGMENTS}

Research of the first author was supported by The Department of National Defence (Canada) Academic Research Program. Research of the second and third authors was supported by Office of Naval Research Grant N00014-92J-1194, Natural Sciences and Engineering Research Council of Canada Grant GPO 105574 and Fonds pour la Formation des Chercheurs et l'Aide à la Recherche Grant 32EQ 1048. Research of the fourth author was supported by an International Postdoctoral Fellowship of the Natural Sciences and Engineering Research Council of Canada, Grant OGPOO 39682. The authors thank Paul Calamai for making the program for his projection method, written with Ingrid Bongartz and Andrew Conn, available to them, and Dominique Peeters for communicating his program for the $p$-Median problem, written with Pierre Hanjoul.

\section{REFERENCES}

Avriel, M. 1976. Nonlinear Programming: Analysis and Methods. Prentice-Hall, Englewood Cliffs, NJ.

Baxter, J. 1981. Local optima avoidance in depot location. $J$. Oper. Res. Soc. 32 815-819.

Bhaskaran, S. 1992. Identification of transshipment center locations. Euro. J. Oper. Res. 63 141-150.

Boese, K. D., A. B. Kahng, S. Muddu. 1994. A new adaptive multi-start technique for combinatorial global optimizations. Oper. Res. Letters 16 101-113.

Bongartz, I., P. H. Calamai, A. R. Conn. 1994. A projection method for $l_{p}$ norm location-allocation problems. Math. Programming 66 283-312.

Brimberg, J., R. Chen, D. Chen. 1998. Accelerating convergence in the Fermat-Weber location problem. Oper. Res. Letters 22 151-157.

— R. F. Love. 1993. Global convergence of a generalized iterative procedure for the minisum location problem with $l_{p}$ distances. Oper. Res. 41 1153-1163. 
-, N. Mladenović. 1996a. Solving the continuous locationallocation problem with Tabu search, Studies in Locational Anal. 8 23-32.

— $-1996 \mathrm{~b}$. A variable neighbourhood algorithm for solving the continuous location-allocation problem. Studies in Locational Anal. 10 1-12.

_, 1998 . Degeneracy in the multi-source Weber problem. Les Cahiers du GERAD, G-98-08, Montreal. Also appears in Math. Programming (1999) 85 213-220.

Charalambous, C., J. W. Bandler. 1976. Non-linear optimization as a sequence of least $p$-th optimization with finite values of p. Internat. J. System Sci. 7 377-391.

Chen, R. 1983. Solution of minisum and minimax locationallocation problems with Euclidean distances. Naval Res. Logist. Quart. 30 449-459.

Chen, P. C., P. Hansen, B. Jaumard, H. Tuy. 1992. Solution of the multisource Weber and conditional Weber problems by d.-c. programming. Les Cahiers du GERAD, G-92-35, Montreal, Canada, Oper. Res. 46 (1998) 548-562.

Cooper, L. 1963. Location-allocation problems. Oper. Res. 11 331-343.

- 1964. Heuristic methods for location-allocation problems. SIAM Rev. 6 37-53.

- 1967. Solutions of generalized locational equilibrium models. J. Regional Sci. 7 1-18.

Drezner, Z. 1984. The planar two-center and two-median problems. Transp. Sci. 18 351-361.

- 1992. A note on the Weber location problem. Ann. Oper. Res. 40 153-161.

Eilon, S., C. D. T. Watson-Gandy, N. Christofides. 1971. Distribution Management. Hafner, New York.

Fleischmann, B., J. Paraschis. 1988. Solving a large scale districting problem: a case report. Comput. Oper. Res. 15 521-533.

Frenk, J. B. G., M. T. Melo, S. Zhang. 1994. A Weiszfeld method for a generalized $l_{p}$ distance minisum location model in continuous space. Location Sci. 2 111-127.

Gendreau, M., A. Hertz, G. Laporte. 1996. The Traveling Salesman Problem with backhauls. Comput. Oper. Res. 23 501-508.

Glover, F. 1989. Tabu search. Part I. ORSA J. Comput. 1 190-206.

—. 1990. Tabu search. Part II. ORSA J. Comput. 2 4-32.

—, M. Laguna. 1993. Tabu search. In Modern Heuristic Techniques for Combinatorial Problems (Chapter 3), C. Reeves (Ed.), Oxford, Blackwell.

Hanjoul. P., D. Peeters. 1985. A comparison of two dual-based procedures for solving the $p$-Median problem. Euro. J. Oper. Res. 20 387-396.

Hansen, P., B. Jaumard. 1990. Algorithms for the maximum satisfiability problem. Comput. 44 279-303.

N. Mladenović. 1997. An introduction to variable neighborhood search. Les Cahiers du GERAD, G-97-51, Montreal, Canada and Metaheuristics Advances and Trends in Local Search Paradigms for Optimization, S. Voss et al. (eds.), Kluwer, Dordrecht, 1999.

,-- É. Taillard. 1996. Heuristic solution of the multisource Weber problem as a $p$-Median problem. Les Cahiers $d u$ GERAD, G-96-10, Montreal, Canada, Oper. Res. Letters 22 (1998) 55-62.

, B. Jaumard, S. Krau, O. du Merle. 1997. A column generation algorithm for the multisource Weber problem. Les Cahiers du GERAD (forthcoming 2000).
Holland, J. H. 1975. Adaptation in Natural and Artificial Systems. The University of Michigan Press, Ann Arbor, MI.

Houck, C. R., J. A. Joines, M. G. Kay. 1996. Comparison of genetic algorithms, random restart and two-opt switching for solving large location-allocation problems. Comput. Oper. Res. 23 587-596.

Krau, S. 1997. Extensions du problème de Weber. Ph.D. Thèse, École Polytechnique de Montréal (under direction of $\mathrm{P}$. Hansen and B. Jaumard).

Kuenne, R. E., R. M. Soland. 1972. Exact and approximate solutions to the multisource Weber problem. Math. Programming 3 193-209.

Kuhn, H. W. 1973. A note on Fermat's problem. Math. Programming 4 98-107.

Love, R. F., H. Juel. 1982. Properties and solution methods for large location-allocation problems. J. Oper. Res. Soc. 33 $443-452$

— J. G. Morris. 1975. A computational procedure for the exact solution of location-allocation problems with rectangular distances. Naval Res. Logist. Quart. 22 441-453.

,-- G. O. Wesolowsky. 1988. Facilities Location: Models and Methods. North Holland, New York.

Megiddo, N., K. J. Supowit. 1984. On the complexity of some common geometric location problems. SIAM J. Comput. 13 182-196.

du Merle, O., D. Villeneuve, J. Desrosiers, P. Hansen. 1996. Stabilization dans le cadre de la génération de colonnes. Les Cahiers du GERAD G-97-08, Montreal.

Mirchandani, P., R. Francis (eds.). 1990. Discrete Location Theory. Wiley-Interscience, New York.

Mladenović, N. 1995. A variable neighbourhood algorithm-a new metaheuristic for combinatorial optimization. Abstract of papers presented at Optimization Days, Montreal, 112-112.

_ J. Brimberg. 1995. A descent-ascent technique for solving the multisource Weber problem. Yugoslav J. Oper. Res. 5 211-219.

_ P. Hansen. 1997. Variable neighbourhood search. Comput. Oper. Res. 24 1097-1100.

Moreno, J., C. Rodrigez, N. Jimenez. 1990. Heuristic cluster algorithm for multiple facility location-allocation problem. RAIRO. Oper. Res. 25 97-107.

Murtagh, B. A., S. R. Niwattisyawong. 1982. An efficient method for the multi-depot location-allocation problem. J. Oper. Res. Soc. 33 629-634.

Ostresh, L. M., Jr. 1973a. TWAIN-exact solutions to the two source location-allocation problem. In Computer Programs for Location-Allocation Problems. G. Rushton, M. F. Goodchild and L. M. Ostresh Jr. (eds.). Monograph Number 6, Department of Geography, University of Iowa, Iowa City, IA. 1973b. MULTI - exact solutions to the $M$-center locationallocation problem. In Computer Programs for LocationAllocation Problems. G. Rushton, M. F. Goodchild and L. M. Ostresh Jr. (eds.). Monograph Number 6, Department of Geography, University of Iowa, Iowa City, IA.

1975. An efficient algorithm for solving the two center location-allocation problem. J. Regional Sci. 15 209-216. 
460 / Brimberg, Hansen, Mladenović, AND Taillard

Reinelt, G. 1991. TSLIB - a traveling salesman library. ORSA J. Comput. 3 376-384.

Rolland, E., D. A. Schilling, J. R. Current. 1996. An efficient Tabu search procedure for the $p$-median problem. Euro. J. Oper. Res. 96 329-342.

Rosen, J. B., G.-L. Xue. 1991. Computational comparison of two algorithms for the Euclidean single facility location problem. ORSA J. Comput. 3 207-212.

Rosing, K. E. 1992. An optimal method for solving the (generalized) multi-Weber problem. Euro. J. Oper. Res. 58 $414-426$
Scott, A. J. 1970. Location-allocation systems: a review. Geographical Anal. 2 95-119.

Sullivan, P. J., N. Peters. 1980. A flexible user oriented locationallocation algorithm. J. Environmental Management 10 181-193.

Teitz, M. B., P. Bart. 1968. Heuristic methods for estimating the generalized vertex median of a weighted graph. Oper. Res. 16 955-961.

Whitaker, R. 1983. A fast algorithm for the greedy-interchange for large-scale clustering and median location problems. INFOR 21 95-108. 